\title{
Pregnancy outcomes in women with kidney transplant: Metaanalysis and systematic review
}

\author{
Silvi Shah ${ }^{1 *} \mathbb{D}$, Renganathan Lalgudi Venkatesan², Ayank Gupta², Maitrik K. Sanghavi², Jeffrey Welge ${ }^{3}$, \\ Richard Johansen², Emily B. Kean², Taranpreet Kaur ${ }^{1}$, Anu Gupta ${ }^{4}$, Tiffany J. Grant ${ }^{2}$ and Prasoon Verma ${ }^{5}$
}

\begin{abstract}
Background: Reproductive function in women with end stage renal disease generally improves after kidney transplant. However, pregnancy remains challenging due to the risk of adverse clinical outcomes.

Methods: We searched PubMed/MEDLINE, Elsevier EMBASE, Scopus, BIOSIS Previews, ISI Science Citation Index Expanded, and the Cochrane Central Register of Controlled Trials from date of inception through August 2017 for studies reporting pregnancy with kidney transplant.

Results: Of 1343 unique studies, 87 met inclusion criteria, representing 6712 pregnancies in 4174 kidney transplant recipients. Mean maternal age was $29.6 \pm 2.4$ years. The live-birth rate was $72.9 \%(95 \% \mathrm{Cl}, 70.0-75.6)$. The rate of other pregnancy outcomes was as follows: induced abortions (12.4\%; 95\% Cl, 10.4-14.7), miscarriages (15.4\%; 95\% Cl, 13.8-17.2), stillbirths (5.1\%; 95\% Cl, 4.0-6.5), ectopic pregnancies (2.4\%; 95\% Cl, 1.5-3.7), preeclampsia (21.5\%; 95\% Cl, 18.5-24.9), gestational diabetes $(5.7 \%$; $95 \% \mathrm{Cl}, 3.7-8.9)$, pregnancy induced hypertension $(24.1 \%$; $95 \% \mathrm{Cl}, 18.1-31.5)$, cesarean section (62.6, 95\% Cl 57.6-67.3), and preterm delivery was 43.1\% (95\% Cl, 38.7-47.6). Mean gestational age was 34.9 weeks, and mean birth weight was $2470 \mathrm{~g}$. The 2-3-year interval following kidney transplant had higher neonatal mortality, and lower rates of live births as compared to $>3$ year, and $<2$-year interval. The rate of spontaneous abortion was higher in women with mean maternal age $<25$ years and $>35$ years as compared to women aged $25-34$ years.

Conclusion: Although the outcome of live births is favorable, the risks of maternal and fetal complications are high in kidney transplant recipients and should be considered in patient counseling and clinical decision making.
\end{abstract}

Keywords: Pregnancy, Kidney transplant, Maternal, Fetal, Outcomes

\section{Background}

Women with end stage renal disease have impaired fertility due to disruption of hypothalamic gonadal axis. Pregnancy is therefore rare in women on dialysis with very low incidence of conception ranging from 0.9 to $7 \%$ [1]. Since there is rapid restoration of fertility, in some cases, within 6 months following transplantation, kidney transplantation offers the best hope to women with end-stage renal disease who wish to become pregnant [2].

\footnotetext{
* Correspondence: silvishah2108@gmail.com

${ }^{1}$ Division of Nephrology Kidney C.A.R.E. Program, University of Cincinnati, 231

Albert Sabin Way, MSB 6112, Cincinnati, OH 45267, USA

Full list of author information is available at the end of the article
}

Pregnancy in a kidney transplant recipient continues to remain challenging due to risk of adverse maternal complications of preeclampsia and hypertension, and risk of adverse fetal outcomes of premature birth, low birth weight, and small for gestational age infants [3]. Additionally, there is risk of side effects from immunosuppressive medication, and risk of deterioration of allograft function [4]. Therefore, preconception counseling, family planning and contraception are pertinent parts of the transplant counseling process.

Data on clinical outcomes of pregnancy in kidney transplant recipients is limited from case reports, single-center studies, and voluntary registries. The usefulness of the voluntary registries is further limited due to underreporting and incomplete data capture [5-8].

(c) The Author(s). 2019 Open Access This article is distributed under the terms of the Creative Commons Attribution 4.0 International License (http://creativecommons.org/licenses/by/4.0/), which permits unrestricted use, distribution, and 
To the best of our knowledge, no comprehensive metanalysis on post-kidney transplant pregnancy outcomes has been performed in the recent years [9]. Since kidney transplant is common in women of child bearing age and most of the data on outcomes of pregnancy comes from these retrospective studies, our metaanalysis is both timely and important. The comprehensive analysis of various worldwide registries, single-center studies, and case series will provide generalizable inferences about post-kidney transplant pregnancy outcomes, and help guide the pregnancy in kidney transplant recipients. The primary goal of this study was to perform a meta-analysis to systematically identify all studies of pregnancy-related outcomes in kidney transplant recipients from all around the world, and estimate pooled incidences of pregnancy outcomes, maternal complications, and fetal complications. The secondary goals were to examine the impact of pregnancy on the kidney allograft loss, allograft rejection, identify ideal maternal age of conception, and determine ideal time of conception between kidney transplant and pregnancy.

\section{Methods}

\section{Data sources and searches}

We performed a systematic review and meta-analyses reported according to PRISMA guidelines for studies exploring incidence and outcomes of pregnancy in women with kidney transplant (Fig. 1). We searched PubMed/MEDLINE, Elsevier EMBASE, Scopus, BIOSIS Previews, ISI Science Citation Index Expanded, and the Cochrane Central Register of Controlled Trials (CENTRAL) from their earliest date of inception through 8/31/ 2017, and abstracts from the annual American Transplant Congresses from 1/1/2013 through 8/31/2017. A health sciences librarian (E.K.) developed database-specific search strategies including a combination of subject headings (MeSH or Emtree) and keywords. The following key search terms were used in strategies specific to each database and organization: pregnancy complications, pregnancy outcome, maternal outcome, fetal outcome, birth outcome, kidney transplant, or renal transplant. A reproducible PubMed search strategy is provided in Additional file 1.

\section{Study selection}

We considered observational studies (prospective cohort, retrospective cohort, and cross-sectional), case series, and case reports (with $n>10$ pregnancies) that explored the pregnancy, maternal, and fetal outcomes among women $\geq 18$ years, and who received a kidney transplant. Studies of patients with multiple organ transplants, studies that analyzed the teratogenic effects of

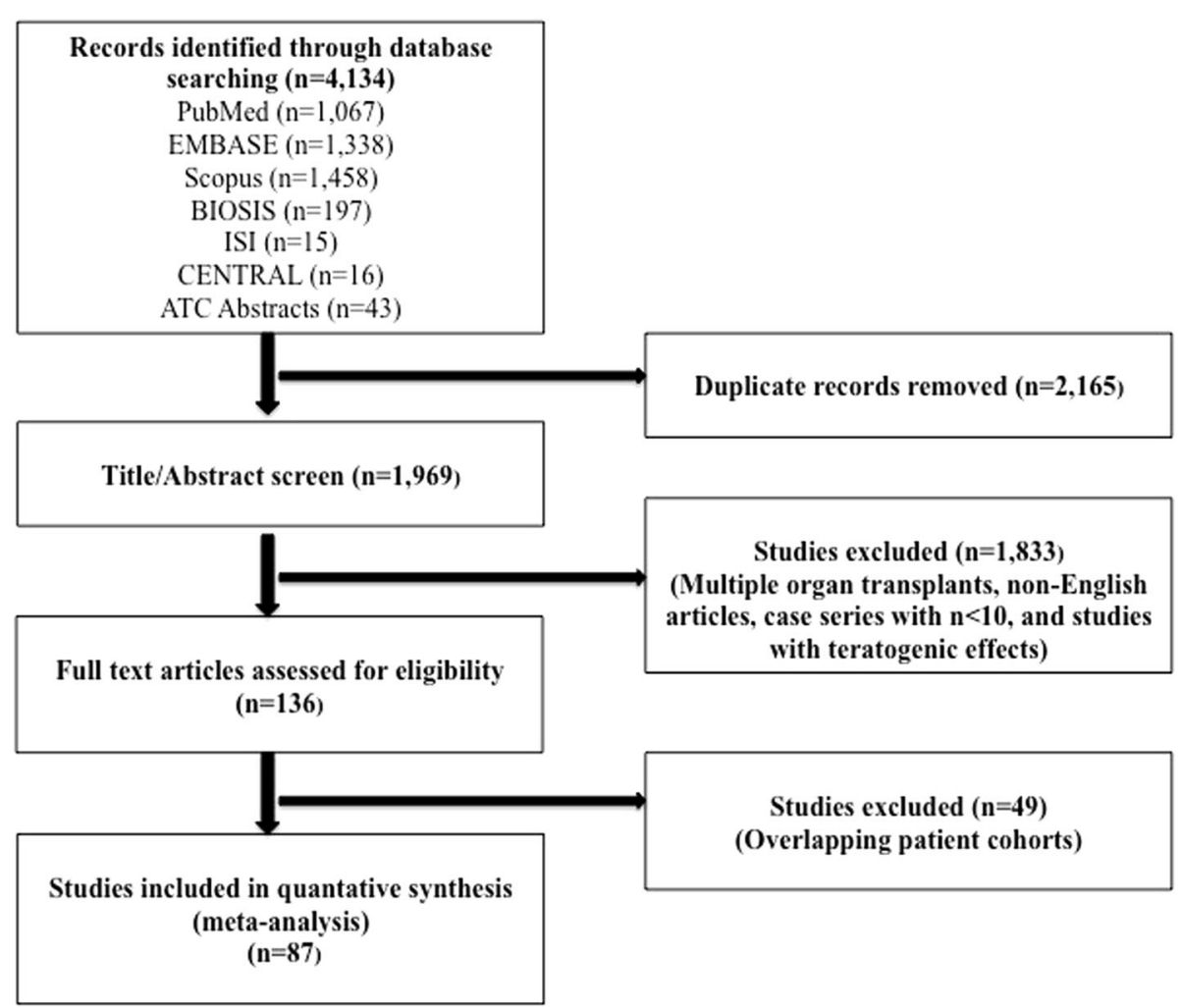

Fig. 1 Study cohort showing the selection of studies reporting outcomes of pregnancies in women with kidney transplant 
mycophenolate or sirolimus, and non-English language studies were excluded. Titles and abstracts of all identified citations were screened independently by two reviewers (S.S. and T.G.), who discarded studies that did not meet all inclusion criteria. The same reviewers independently screened the abstracts of all eligible studies. If eligibility was indeterminable from the abstract, the study was included in the full-text screen. All disagreements were adjudicated by the principal investigator (S.S).

\section{Data extraction, quality assessment, and outcomes}

Data extraction was carried out independently by three data extraction team members (A.G, L.R. and M.S.) using standard data extraction forms. Data elements were then rechecked for accuracy by all the three data extraction team members. When more than one publication of a similar patient population existed with more than $25 \%$ overlap, publication with higher number of pregnancy events and the most complete details was included. Disagreements in data extraction and quality assessment were resolved in consultation with an arbitrator (T.G.) and primary investigator (S.S.). For each included study, the following data was extracted: country of location, years of data collection, number of kidney transplant recipients, number of pregnancies, mean maternal age, mean interval between kidney transplant and pregnancy, pregnancy outcomes (number of live births, miscarriage, induced abortion, still birth and ectopic pregnancies), maternal outcomes (number of women with preeclampsia, pregnancy induced hypertension, and gestational diabetes mellitus, and number of cesarean sections), fetal outcomes (number of pre-term births, mean gestational age, mean gestational weight, and number of neonatal deaths); and graft outcomes (number of acute rejection during pregnancy, graft failure post pregnancy, mean serum creatinine pre and post pregnancy). To maintain consistency across extracted data, the number of pregnancies was used as a denominator for the outcomes of live births, miscarriages, induced abortions, stillbirths, neonatal deaths, preeclampsia, pregnancy induced hypertension, and gestational diabetes mellitus. The number of live births was used as the denominator for the outcome of preterm deliveries, and cesarean section. Preterm was defined as babies born alive before 37 weeks gestation.

\section{Data synthesis and analysis}

Patients characteristics were reported as frequencies. The pregnancy incidence was reported for women per 1000 live births. For each study, estimates were expressed as prevalence and 95\% confidence intervals (CI). Prevalence estimates from individual studies were pooled using a random-effects model. Heterogeneity across included studies was analyzed formally using
Cochran Q (heterogeneity 2) and I2 statistics. For binary outcomes, the DerSimonian-Laird method was used, and for continuous outcomes, a weighted average methodology was used to calculate the pooled estimates and 95\% CI. Two-sample test of proportions was used to compare the pooled incidence for each analysis to the most recent United States (US) general population incidence. [10-14] We determined the associations of maternal age, the interval between kidney transplant, and the pregnancy outcomes. Additonally, we performed a subgroup analysis for the pregnancy, maternal and fetal outcomes for studies published from 2000 to 2017. Analyses were performed using MS Excel and Comprehensive Meta-analysis packages in $\mathrm{R}$ software.

\section{Results}

Among the 4134 citations that were retrieved, 136 full-text articles were reviewed and 87 were selected to be included in the final study cohort (Fig. 1). Three studies were from Africa, 31 from Asia, 31 from Europe, 10 from North America, 4 from Oceania, and 8 from South America (Table 1). Overall, there were 6712 pregnancies in 4174 kidney transplant recipients. Mean maternal age was $29.6 \pm 2.4$ years and mean interval between kidney transplant and pregnancy was 3.7 years.

\section{Pregnancy outcomes}

Live birth rate was $72.9 \%$ (95\% CI, 70.0-75.6), miscarriages rate was $15.4 \%$ (95\% CI, 13.8-17.2), induced abortions rate was $12.4 \%$ (95\% CI, 10.4-14.7), stillbirths rate was $5.1 \%$ (95\% CI, 4.0-6.5) and rate of ectopic pregnancies was $2.4 \%$ (95\% CI, 1.5-3.7). In our study cohort of kidney transplant recipients, live birth rates were higher as compared to the US general population $(72.9 \%$ vs. $62 \%$ ) and favorable across all geographic regions (Fig. 2) $[10,11]$. Overall, miscarriage rate was slightly lower than that of the US general population ( $15.4 \%$ vs. $17.1 \%)$, but higher across Africa (21.0\%; 95\% CI, 14.3-29.9), and South America (20.2\%; 95\% CI, 15.6-25.7) (Fig. 3) [13]. Induced abortion rate was also lower than the US general population $(12.4 \%$ vs. $18.6 \%)$ [13]. The rate of induced abortion was highest in South America (19.8\%; 95\% CI, 12.2-30.3), followed by Asia (13.3\%; 95\% CI, 9.6-18.3), Oceania (11.5\%; 95\% CI, 9.3-14.0), North America (10.9\%; 95\% CI, 5.9-19.2), Europe (10.0\%; 95\% CI, 7.3-13.5), and Africa (7.7, 95\% CI, 1.4-32.6) (Fig. 4). Overall, stillbirth rate was higher than the US general population (5.1\% vs. 0.6\%) [14]. Worldwide, stillbirth rate was highest in Asia (6.6, 95\% CI, 4.8-9.0\%), and lowest in Africa (2.6, 95\% CI; 0.4-16.5) (Fig. 5). The rate of ectopic pregnancy was slightly higher than the US general population $(2.4 \%$ vs. $1.4 \%)$, with highest rate in Asia (3.3, 95\% CI; 1.1-9.8) (Fig. 6) [15]. The results from the subgroup analyses (2000-2017) for pregnancy 
Table 1 Studies included in the metaanalysis

\begin{tabular}{|c|c|c|c|c|c|}
\hline & Reference, Year Published & Study Years & Country & Recipients & Pregnancies \\
\hline 1 & Devresse et al., 2017 [32] & 1994-2010 & Belgium & 32 & 57 \\
\hline 2 & Yuksel et al., 2017 [33] & 2009-2016 & Turkey & 25 & na \\
\hline 3 & Ajaimy et al., 2016 [34] & 2009-2014 & USA & 11 & 11 \\
\hline 4 & Candido et al., 2016 [35] & 2004-2014 & Portugal & 36 & 53 \\
\hline 5 & Cristelli et al., 2016 [36] & 2004-2014 & Brazil & 36 & 53 \\
\hline 6 & El Houssni et al., 2016 [37] & na & Saudi Arabia & 12 & 21 \\
\hline 7 & Lima et al., 2016 [38] & 2004-2014 & Brazil & 36 & 53 \\
\hline 8 & Majak et al., 2016 [39] & 1969-2013 & Norway & na & 119 \\
\hline 9 & Mishra et al., 2016 [40] & 2004-2014 & India & 16 & na \\
\hline 10 & Orihuela et al., 2016 [41] & $1986-2014$ & Uruguay & 32 & 40 \\
\hline 11 & Piccoli et al., 2016 [42] & 1978-2013 & Italy & na & 189 \\
\hline 12 & Saliem et al., 2016 [43] & $2006-2011$ & Canada & na & 264 \\
\hline 13 & Santos et al., 2016 [44] & 2010-2014 & Portugal & 8 & 8 \\
\hline 14 & Sarween et al., 2016 [45] & $2001-2015$ & UK & 387 & 569 \\
\hline 15 & Stoumpos et al., 2016 [46] & 1973-2013 & UK & 89 & 138 \\
\hline 16 & Yoshikawa et al., 2016 [47] & na & Japan & 49 & 65 \\
\hline 17 & Aktrurk et al., 2015 [48] & 2004-2014 & Turkey & 12 & 16 \\
\hline 18 & Arab et al., 2015 [49] & $2003-2010$ & Canada & na & 375 \\
\hline 19 & Erman et al., 2015 [50] & $1987-2011$ & Turkey & 43 & 43 \\
\hline 20 & Yeon et al., 2015 [51] & $1995-2015$ & Korea & 84 & 119 \\
\hline 21 & Debska - Slizien et al., 2014 [52] & 1980-2012 & Poland & 17 & 22 \\
\hline 22 & Farr et al., 2014 [53] & 1999-2013 & Austria & 12 & 12 \\
\hline 23 & Hebral et al., 2014 [54] & 1969-2011 & France & 46 & 61 \\
\hline 24 & You et al., 2014 [55] & 1995-2013 & Korea & 29 & 41 \\
\hline 25 & Blume et al., 2013 [56] & 1988-2010 & Germany & 34 & 53 \\
\hline 26 & Guella et al., 2013 [57] & 1992-2008 & Saudi Arabia & 15 & 33 \\
\hline 27 & Pietrzak et al., 2013 [58] & $2001-2012$ & Poland & 34 & 40 \\
\hline 28 & Rachdi et al., 2013 [59] & 2003-2013 & Tunisia & 12 & 17 \\
\hline 29 & Ribeiro et al., 2013 [60] & 1995-2007 & Brazil & 22 & 31 \\
\hline 30 & Rocha et al., 2013 [61] & 1983-2009 & Portugal & 24 & 25 \\
\hline 31 & Wyld et al., 2013 [62] & $1971-2010$ & Australia & 447 & 692 \\
\hline 32 & Kennedy et al., 2012 [63] & na & Ireland & 18 & 29 \\
\hline 33 & Neyatani et al., 2012 [64] & $1975-2011$ & Japan & 22 & 34 \\
\hline 34 & Van Buren et al., 2012 [65] & $1971-2010$ & Netherlands & 30 & 42 \\
\hline 35 & Celik et al., 2011 [66] & 1998-2008 & Turkey & 24 & 31 \\
\hline 36 & Gerlei et al., 2011 [67] & 1974-2010 & Hungary & 23 & 27 \\
\hline 37 & Lopez et al., 2011 [68] & $1986-2010$ & Spain & 20 & 24 \\
\hline 38 & Xu et al., 2011 [69] & 1989-2008 & China & 25 & 38 \\
\hline 39 & Gorgulu et al., 2010 [70] & 1983-2008 & Turkey & 19 & 22 \\
\hline 40 & Areia et al., 2009 [71] & 1989-2007 & Portugal & 28 & 34 \\
\hline 41 & Gill et al., 2009 [20] & 1990-2003 & USA & 483 & 530 \\
\hline 42 & Levidiotis et al., 2009 [8] & $1966-2005$ & Australia & 381 & 577 \\
\hline 43 & Rizvi et al., 2009 [72] & $1985-2008$ & Pakistan & 45 & 72 \\
\hline 44 & Sharma et al., 2009 [73] & 1988-2006 & Oman & 42 & 82 \\
\hline
\end{tabular}


Table 1 Studies included in the metaanalysis (Continued)

\begin{tabular}{|c|c|c|c|c|c|}
\hline & Reference, Year Published & Study Years & Country & Recipients & Pregnancies \\
\hline 45 & Al Duraihimh et al., 2008 [74] & 1996-2006 & Middle East & 140 & 234 \\
\hline 46 & Alfi A Yet al, 2008 [75] & 1989-2005 & Saudi Arabia & 12 & 20 \\
\hline 47 & Cruz Lemini et al., 2007 [28] & 1990-2005 & Mexico & 60 & 75 \\
\hline 48 & Oliveira et al., 2007 [76] & $2001-2005$ & Brazil & 52 & 52 \\
\hline 49 & Sibanda et al., 2007 [77] & 1994-2001 & UK & 176 & 193 \\
\hline 50 & Yassaee et al., 2007 [78] & 1996-2001 & Iran & 74 & 95 \\
\hline 51 & Kurata et al., 2006 [79] & $1984-2003$ & Japan & 42 & 53 \\
\hline 52 & Rahamimov et al., 2006 [80] & 1983-1998 & Israel & 39 & 69 \\
\hline 53 & Galdo et al., 2005 [81] & 1982-2002 & Chile & 30 & 37 \\
\hline 54 & Garcia - Donaire et al., 2005 [82] & 1997-2004 & Spain & 16 & 19 \\
\hline 55 & Ghanem et al., 2005 [83] & 1989-2004 & Egypt & 41 & 67 \\
\hline 56 & Pour-Reza-Gholi et al., 2005 [84] & $1984-2004$ & Iran & 60 & 74 \\
\hline 57 & Yildirim et al., 2005 [85] & 1998-2005 & Turkey & 17 & 20 \\
\hline 58 & Keitel et al., 2004 [86] & 1977-2001 & Brazil & 41 & 44 \\
\hline 59 & Pezeshki et al., 2004 [87] & $1991-1998$ & Iran & 18 & 20 \\
\hline 60 & Hooi et al., 2003 [88] & 1984-2001 & Malaysia & 46 & 72 \\
\hline 61 & Queipo et al., 2003 [89] & $1980-2000$ & Spain & 29 & 40 \\
\hline 62 & Thompson et al., 2003 [90] & $1976-2001$ & UK & 24 & 48 \\
\hline 63 & Sgro et a,l 2002 [91] & 1988-1998 & Canada & 26 & 44 \\
\hline 64 & Tan et al., 2002 [92] & $1986-2000$ & Singapore & 25 & 42 \\
\hline 65 & Park et al., 2001 [93] & na - 2000 & South Korea & 36 & 47 \\
\hline 66 & Kuvacic et al., 2000 [94] & 1986-1996 & Croatia & 15 & 23 \\
\hline 67 & Little et al., 2000 [27] & 1985-1998 & Ireland & 19 & 29 \\
\hline 68 & Moon et al., 2000 [95] & na - 1998 & Korea & 36 & 48 \\
\hline 69 & Ventura et al., 2000 [96] & 1983-1999 & Portugal & 15 & 15 \\
\hline 70 & Arsan et al., 1997 [97] & na & France & 20 & 33 \\
\hline 71 & Rahbar et al., 1997 [98] & 1985-1993 & Iran & 13 & 14 \\
\hline 72 & Rieu et al., 1997 [99] & 1970-1995 & France & 22 & 33 \\
\hline 73 & Al Hassani et al., 1995 [100] & 1985-1993 & Oman & 25 & 44 \\
\hline 74 & Sabagh et al., 1995 [101] & 1984-1994 & Saudi Arabia & 33 & 52 \\
\hline 75 & Saber et al., 1995 [102] & 1968-1992 & Brazil & 19 & 25 \\
\hline 76 & Wong et al., 1995 [103] & 1972-1992 & New Zealand & 9 & 16 \\
\hline 77 & Hadi et al., 1986 [104] & 1969-1992 & South Korea & 11 & 13 \\
\hline 78 & Talaat et al., 1994 [105] & 1977-1992 & Sweden & 19 & 25 \\
\hline 79 & Pahl et al., 1993 [106] & 1969-1990 & USA & 21 & 32 \\
\hline 80 & Muirhead et al., 1992 [107] & 1977-1988 & UK & 22 & 22 \\
\hline 81 & Brown et al., 1991 [108] & 1965-1989 & Ireland & 14 & 27 \\
\hline 82 & Sturgiss et al., 1991 [109] & 1967-1987 & UK & 17 & 22 \\
\hline 83 & O' Connell et al., 1989 [110] & 1974-1986 & Australia & 11 & 18 \\
\hline 84 & Ha et al., 1994 [111] & 1970-1982 & USA & 13 & 17 \\
\hline 85 & Marushak et al., 1986 [112] & 1972-1983 & Denmark & 20 & 24 \\
\hline 86 & O' Donnell et al., 1985 [113] & 1971-1984 & South Africa & 21 & 38 \\
\hline 87 & Waltzer et al., 1980 [114] & na & USA & 12 & 15 \\
\hline
\end{tabular}




\section{Live Births among Kidney Transplant Recipients}

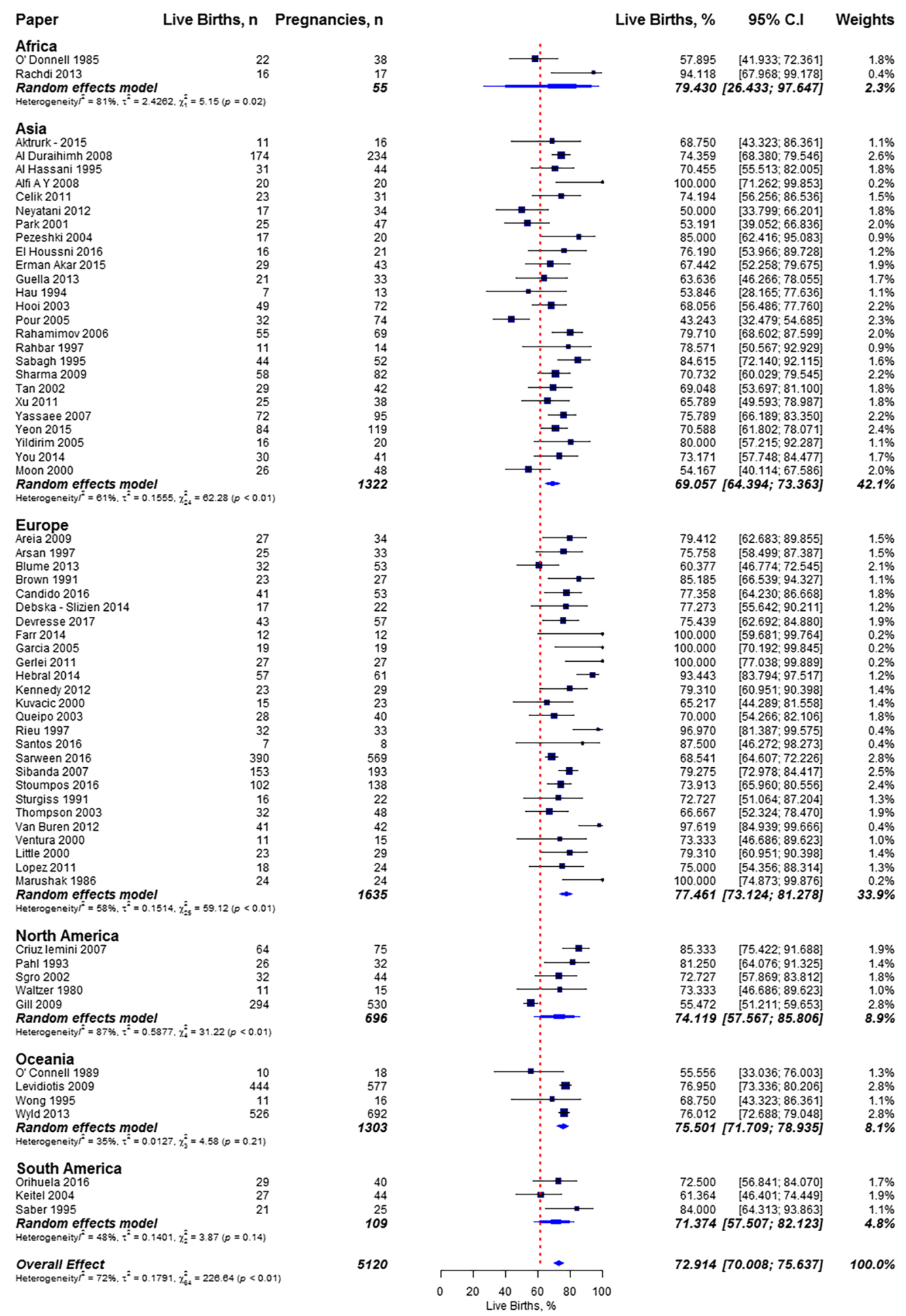

Fig. 2 Forest Plot showing outcome of live births among kidney transplant recipients overall, and across different geographical regions 
Miscarriage among Kidney Transplant Recipients

Paper

Africa

O'Donnell 1985

Ghanem 2005

Random effects mode

Heterogeneity $t^{2}=0 \%, \tau^{2}=0 . \%_{4}^{2}=0.27(p=0.80$

Asia

Aktrurk - 2015

Al Durainimh 2008

Al Hassani 1995

Alfi A Y 2008

Neyatani 2012

Park 2001

Pezeshki 2004

Erman Akar 2015

Hooi 2003

Rahbar 1997

Rizvi 2009

Sabagh 1995

Sharma 200

Tan 2002

Xu 2011

Yassaee 2007

Yeon 2015

Yildirim 2005

Moon 2000

Random effects mode

Heterogeneity $l^{2}=0 \%, z^{2}=0 . \dot{\psi}_{13}^{2}=17.79(p=0.47)$

Miscarriage, $n$ Pregnancies, $n$

Europe

Areia 2009

Arsan 1997

Blume 2013

Candido 2016

Devresse 2017

Hebral 2014

Kennedy 2012

Sarween 2016

Stoumpos 2016

Little 2000

Lopez 2011

Random effects mode

Heterogeneity $l^{2}=20 \% . \tau^{2}=0.0255 . \hat{x_{13}}=18.21(p=0.24)$

North America

Ajaimy 2016

Criuz lemini 2007

Pahl 1993

Sgro 2002

Waltzer 1980

Gill 2009

Random effects mode

Heterogeneity $I^{2}=15 \%, \tau^{2}=0.0293 . \psi_{.5}^{2}=5.87(p=0.32)$

\section{Oceania}

O' Connell 1989

Wong 1995

Wyld 2013

Random effects model

Heteroneneity $1^{2}=4 \%, z^{2}=0.0138, \hat{\prime}=2.09(p=0.35)$

South America

Cristelli 2016

Orihuela 201

Galdo 2005

Keitel 2004

Saber 1995

Lima 2016

Random effects model
Heterogeneity $I^{2}=0 \%,:^{2}=0 . \psi_{5}^{*}=4.25(p=0.51)$

Overall Effect

Heterogeneityl ${ }^{2}=38 \% . \tau^{2}=0.0578 . \hat{x_{49}}=78.95(p<0.01)$
Miscarriage, $\%$

$95 \%$ C.I

Weights

$23.684 \quad[12.809 ; 39.599] \quad 2.1 \%$

$19.403 \quad[11.614 ; 30.608] \quad 2.8 \%$

21.024 [14.257; 29.883] $\quad 4.9 \%$

$25.000 \quad[9.707 ; 50.824] \quad 1.1 \%$

$19.231 \quad[14.677 ; 24.787] \quad 5.0 \%$

$11.364 \quad[4.810 ; 24.544] \quad 1.5 \%$

$0.000 \quad[0.147 ; 28.738] \quad 0.2 \%$

$23.529 \quad[12.227 ; 40.462] \quad 1.9 \%$

$8.511 \quad[3.231 ; 20.581] \quad 1.3 \%$

$5.000 \quad[0.700 ; 28.220] \quad 0.4 \%$

$23.256 \quad[12.995 ; 38.074] \quad 2.3 \%$

$15.278 \quad[8.667 ; 25.522] \quad 2.6 \%$

$7.143 \quad[0.996 ; 37.028] \quad 0.4 \%$

$12.500 \quad[6.634 ; 22.314] \quad 2.3 \%$

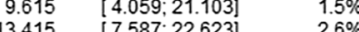

$13.415 \quad[7.587,22.623] \quad 2.6 \%$

[8.667 $[8.159 ; 31.047] \quad 1.9 \%$

$\begin{array}{lll}18.421 & {[9.044 ; 33.897]} & 1.8 \% \\ 16.842 & {[10.582 ; 25.740]} & 3.2 \%\end{array}$

$\begin{array}{lll}16.842 & {[10.582 ; 25.740]} & 3.2 \% \\ 13.445 & {[8.403 ; 20.826]} & 3.3 \%\end{array}$

$5.000 \quad[0.700 ; 28.220] \quad 0.4 \%$

$16.667 \quad[8.560 ; 29.936] \quad 2.0 \%$

$15.983[13.890 ; 18.324] \quad 35.5 \%$

$8.824 \quad[2.874: 24.043]$

$15.152 \quad[6.450 ; 31.622] \quad 1.4 \%$

$24.528 \quad[14.809 ; 37.796] \quad 2.7 \%$

$6.981 \quad[9.079 ; 29.527] \quad 2.2 \%$

$9.091 \quad[2.284: 29.963] \quad 0.7 \%$

$15.789 \quad[8.425 ; 27.647] \quad 2.2 \%$

$1.639 \quad[0.230 ; 10.735] \quad 0.4 \%$

$\begin{array}{lll}13.793 & {[5.275 ; 31.494]} & 1.2 \%\end{array}$

$18.102 \quad[15.150 ; 21.483] \quad 6.1 \%$

$3.9 \%$

$16.667 \quad[11.333 ; 23.835] \quad 3.9 \%$

$\begin{array}{lll}12.500 & {[5.725 ; 25.152]} & 1.7 \% \\ 13.793 & {[5.275 ; 31.494]} & 1.2 \%\end{array}$

$\begin{array}{lll}13.793 & {[5.275 ; 31.494]} & 1.2 \% \\ 16.667 & {[6.399 ; 36.914]} & 1.2 \%\end{array}$

15.493 [13.054; 18.292] $29.8 \%$

$18.182 \quad[4.581 ; 50.702] \quad 0.6 \%$

$13.333 \quad[7.327 ; 23.040] \quad 2.5 \%$

$3.125 \quad[0.438 ; 19.113] \quad 0.4 \%$

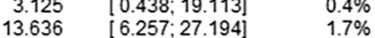

$\begin{array}{lll}13.036 & {[0.257 ; 27.194]} & 1.7 \% \\ 17.358 & {[0.193 ; 35.028]} & 0.2 \%\end{array}$

$17.358 \quad[14.366 ; 20.823] \quad 6.0 \%$

15.202 [11.537; 19.773] $11.4 \%$

$5.556 \quad[0.777 ; 30.652] \quad 0.4 \%$

$6.170,44.746$

$9.056[6.658 ; 12.206] \quad 6.9 \%$

$22.642 \quad[13.332 ; 35.770] \quad 2.6 \%$

$25.000 \quad[14.012 ; 40.542] \quad 2.2 \%$

$18.919 \quad[9.296 ; 34.692] \quad 1.8 \%$

$13.636 \quad[6.257 ; 27.194] \quad 1.7 \%$

$22.642 \quad[13.332 .35 .770] \quad 26 \%$

20.152 [15.557; 25.693] $11.6 \%$

$15.412[13.819 ; 17.151] \quad 100.0 \%$

Fig. 3 Forest Plot showing outcome of miscarriages among kidney transplant recipients overall, and across different geographical regions 
Abortions among Kidney Transplant Recipients

Paper

Africa

O' Donnell 1985

Random effects model

Heterogeneity $\hat{r}=78 \%, z^{2}=1.2785, \hat{z}=4.58(p=0.03)$

Asia

Aktrurk - 2015

Al Hassani 1995

Alfi A Y 2008

Neyatani 2012

Park 2001

El Houssni 2016

Gorgulu 2010

Guella 2013

Hau 1994

Hooi 2003

Pour 2005

Rahamimov 2006

Rahbar 1997

Rizvi 2009

Sabagh 1995

Sharma 2009

Tan 2002

Xu 2011

Yassaee 2007

Yeon 2015

Yildirim 2005

You 2014

Moon 2000

Random effects mode

Abortions, $\mathbf{n}$ Pregnancies, $\mathbf{n}$

10

38
67
105

$=$

16

Europe

Areia 2009

Arsan 1997

Blume 2013

Brown 1991

Debska - Slizien 2014

Devresse 2017

Garcia 2005

Hebral 2014

Kuvacic 2000

Queipo 2003

Sarween 2016

Sibanda 2007

Stoumpos 2016

Ventura 2000

Random effects mode

Heterogeneity $\tilde{r}^{2}=54 \%, \tau^{2}=0.2088, z_{15}^{\hat{z}}=32.57(p<0.01)$

North America

Criuz lemini 2007

Pahl 1993

Hadi 1986

Sgro 2002

Waltzer 1980

Muirhead 1992

Gill 2009

Random effects model

Heterogeneity $\tilde{r}^{\hat{2}}=59 \%, \tau^{2}=0.3958, \gamma_{0}^{2}=14.67(p=0.02)$

Oceania

O' Connell 1989

Wong 1995

Wyld 2013

Random effects model

Heterogeneity $\hat{f}^{2}=0 \%, \tau^{2}=0 . \dot{x}_{\hat{z}}^{*}=0.51(p=0.77)$

South America

Keitel 2004

Ribeiro 2013

Random effects mode

2

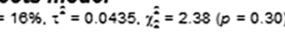

Overall Effect

Heterogeneity $\hat{r}^{2}=65 \%, \tau^{2}=0.2809, \hat{\psi_{.53}}=151.14(p<0.01)$
Abortions, \%

\section{Abortions,}

$95 \%$ C.I Weights

$\begin{array}{rrr}15.789 & {[7.270 ; 30.959]} & 2.2 \% \\ 2.985 & {[0.748 ; 11.163]} & 1.3 \% \\ \mathbf{7 . 6 5 8} & {[\mathbf{1 . 4 0 3 ;} \mathbf{3 2 . 5 8 4}]} & \mathbf{3 . 5 \%}\end{array}$

$0.000 \quad[0.181 .33 .559]$

$6.818 \quad[2.216 ; 19.113] \quad 1.6 \%$

$0.000 \quad[0.147 ; 28.738] \quad 0.4 \%$

$\begin{array}{lll}26.471 & {[14.387 ; 43.542]} & 2.5 \% \\ 38.298 & {[25.635 ; 52.777]} & 2.9 \%\end{array}$

$\begin{array}{lll}38.298 & {[25.635 ; 52.777]} & 2.9 \% \\ 14.286 & {[4.680 ; 36.135]} & 1.6 \%\end{array}$

$0.000 \quad[0.135 ; 26.811] \quad 0.4 \%$

$\begin{array}{lll}30.303 & {[17.146 ; 47.739]} & 2.5 \% \\ 38.462 & {[16.976 ; 65.641]} & 1.7 \%\end{array}$

$\begin{array}{lll}38.462 & {[16.976,65.641]} & 1.7 \% \\ 12.500 & {[6.634 ; 22.314]} & 2.6 \%\end{array}$

$24.324 \quad[15.895 ; 35.344] \quad 3.0 \%$

$20.290 \quad[12.401 ; 31.398] \quad 2.9 \%$

$\begin{array}{lll}7.143 \quad[0.996 ; 37.028] & 0.8 \%\end{array}$

$\begin{array}{lll}6.944 & {[2.920 ; 15.622]} & 2.1 \% \\ 3.846 & {[0.964 ; 14.118]} & 1.3 \%\end{array}$

$\begin{array}{lll}6.098 & {[2.561 ; 13.826]} & 2.1 \%\end{array}$

$7.143 \quad[2.322 ; 19.930] \quad 1.6 \%$

$15.789 \quad[7.270 ; 30.959] \quad 2.2 \%$

$5.263 \quad[2.208 ; 12.027] \quad 2.2 \%$

$\begin{array}{rrr}9.244 & {[5.193 ; 15.924]} & 2.8 \% \\ 15.000 & {[4.917 ; 37.584]} & 1.6 \%\end{array}$

$\begin{array}{rrr}15.000 & {[4.917 ; 37.584]} & 1.6 \% \\ 4.878 & {[1.223 ; 17.517]} & 1.3 \%\end{array}$

$\begin{array}{rrr}29.167 & {[18.098 ; 43.416]} & 2.8 \% \\ 13.373 & {[9.608 ; 18.315]} & 43.6 \%\end{array}$

$\begin{array}{rrr}5.882 & {[1.476 ; 20.685]} & 1.3 \% \\ 9.091 & {[2.962 ; 24.680]} & 1.6 \% \\ 13.208 & {[6.429 ; 25.208]} & 2.4 \% \\ 14.815 & {[5.673 ; 33.461]} & 1.8 \% \\ 4.545 & {[0.636 ; 26.145]} & 0.8 \% \\ 3.509 & {[0.879 ; 12.973]} & 1.3 \% \\ 0.000 & {[0.155 ; 29.808]} & 0.4 \% \\ 0.000 & {[0.111 ; 22.962]} & 0.4 \% \\ 4.918 & {[1.595 ; 14.169]} & 1.7 \% \\ 26.087 & {[12.216 ; 47.234]} & 2.1 \% \\ 22.500 & {[12.144 ; 37.880]} & 2.5 \% \\ 13.357 & {[10.800 ; 16.407]} & 3.7 \% \\ 5.699 & {[3.184 ; 9.997]} & 2.8 \% \\ 5.797 & {[2.925 ; 11.164]} & 2.6 \% \\ 12.500 & {[5.725 ; 25.152]} & 2.3 \% \\ 13.333 & {[3.355 ; 40.538]} & 1.2 \% \\ 9.979 & {[7.282 ; 13.529]} & \mathbf{2 9 . 0 \%}\end{array}$

$\begin{array}{rrr}1.333 & {[0.188 ; 8.860]} & 0.8 \% \\ 9.375 & {[3.055 ; 25.350]} & 1.6 \% \\ 23.529 & {[9.118 ; 48.550]} & 1.7 \% \\ 4.545 & {[1.140 ; 16.438]} & 1.3 \% \\ 26.667 & {[10.377 ; 53.314]} & 1.7 \% \\ 0.000 & {[0.135 ; 26.811]} & 0.4 \% \\ 14.340 & {[11.606 ; 17.589]} & 3.7 \% \\ 10.911 & {[\mathbf{5 . 9 2 6 ; 1 9 . 2 3 3 ]}} & \mathbf{1 1 . 3 \%}\end{array}$

$16.667 \quad[5.473 ; 40.858] \quad 1.5 \%$

$12.500 \quad[3.145 ; 38.596] \quad 1.2 \%$

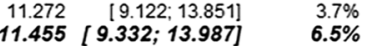

$\begin{array}{lll}22.727 & {[12.688 ; 37.316]} & 2.6 \% \\ 22.581 & {[11.164 ; 40.367]} & 2.3 \%\end{array}$

$\begin{array}{rr}22.581 \quad[11.164 ; 40.367] & 2.3 \% \\ 8.000 \quad[2.009 ; 26.944] & 1.3 \%\end{array}$

$\begin{array}{rr}19.754 \\ {[12.233 ; 30.303]} & \mathbf{6 . 2} \%\end{array}$

12.388 [10.396; 14.699$] \quad 100.0 \%$

Fig. 4 Forest Plot showing outcome of induced abortions among kidney transplant recipients overall, and across different geographical regions 


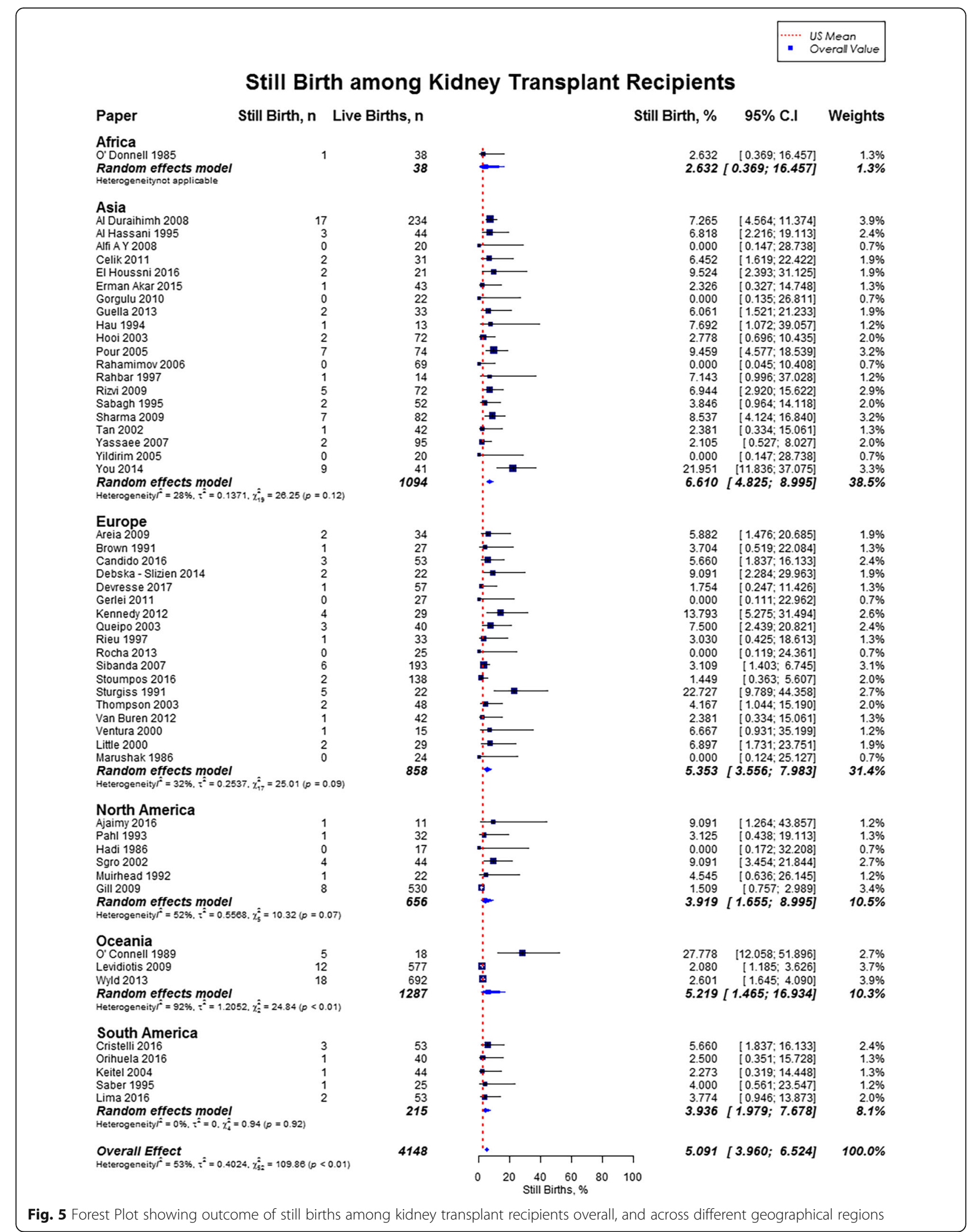




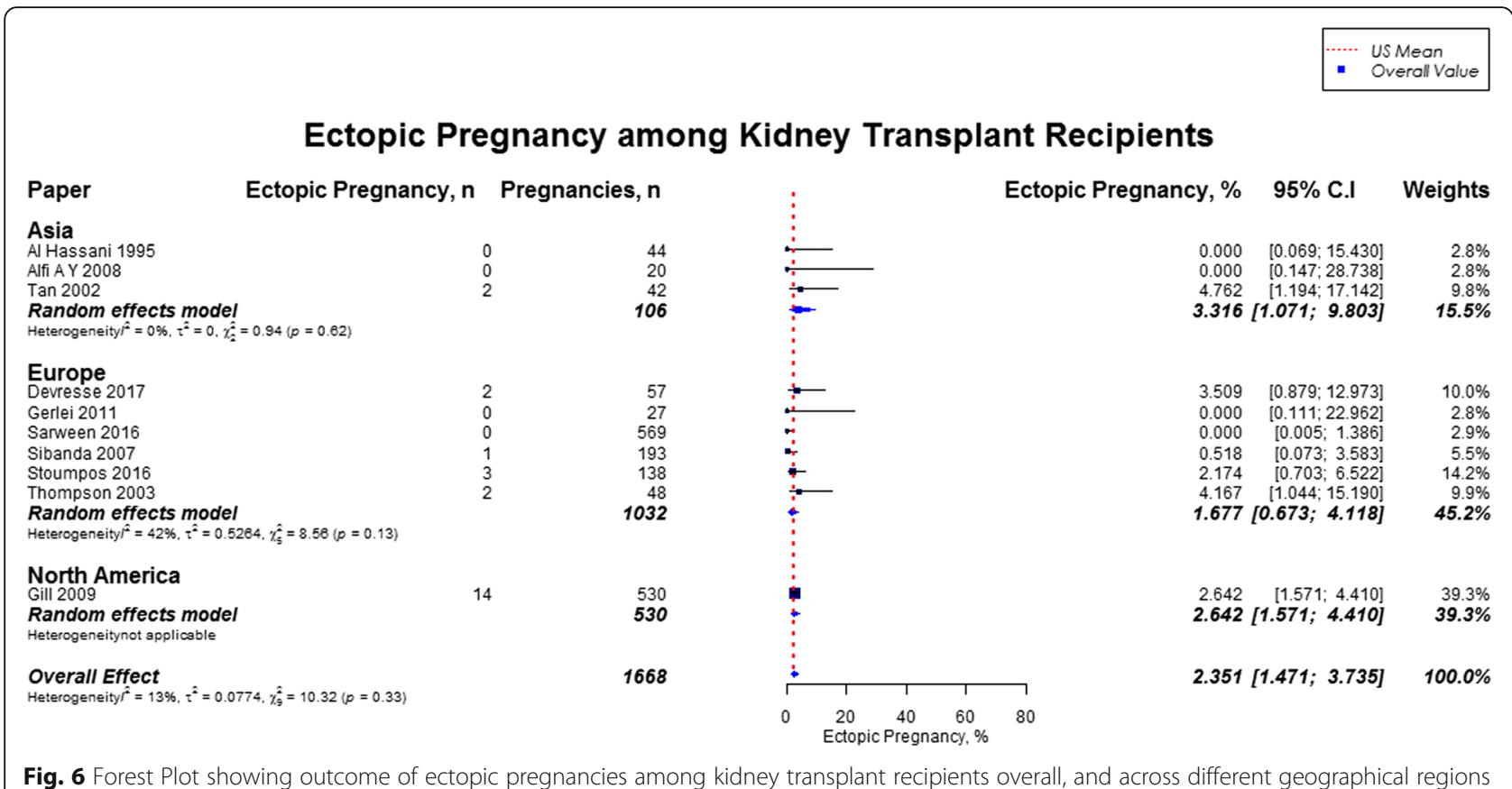

outcomes were consistent with the current findings (Additional file 2).

\section{Maternal outcomes}

Overall, rates of preeclampsia was $21.5 \%$ (95\% CI, 18.524.9; US mean, 3.8\%), cesarean section was $62.6 \%$ (95\% CI, 57.6-67.3; US mean, 31.9\%), gestational diabetes was 5.7\% (95\% CI, 3.7-8.9; US mean, 9.2\%), and pregnancy induced hypertension was $24.1 \%$ (95\% CI, 18.1-31.5). $[12,16]$ Preeclampsia rate was highest in Oceania (27.0\%; 95\% CI, 23.6-30.8), followed by North America (25.5\%; 95\% CI, 14.5-40.8), and lowest in Africa (10.5\%; 95\% CI, 4.0-24.9\%) (Fig. 7). Cesarean section rate was highest in South America (88.8\%; 95\% CI, 49.3-98.5), followed by Africa (77.5\%; 95\% CI, 6.3-99.4) (Fig. 8). Worldwide, Oceania had the lowest rates of gestational diabetes (1.0\%; 95\% CI, 0.5-2.3\%) (Fig. 9). With regards to pregnancy induced hypertension, highest rate was reported in South America (48.0, 95\% CI, 15.1-82.7), while lowest rate was in Africa (16.1, 95\% CI, 9-26.9) (Fig. 10). The results from the subgroup analyses (2000-2017) for maternal outcomes were consistent with the current findings (Additional file 2).

\section{Fetal outcomes}

Overall, rate of preterm birth was 43.1\% (95\% CI, 38.747.6) defined by babies born alive before 37 weeks of gestation, and neonatal mortality was 3.8\% (95\% CI, 2.8-5.2). Rates of preterm birth was highest in South America (55.0\%), and lowest in North America (35.4\%) (Fig. 11). The mean gestational age for newborns was 34.9 weeks
(US mean, 38.7 weeks) and the mean birth weight was 2470 g (US mean, 3389 g). [12, 17] Neonatal mortality was high across all geographical regions as compared to the US mean (3.8\% vs. $0.4 \%)$, with highest rate in Africa (18.4\%; 95\% CI, 9.1-33.9) and lowest rate in North America (1.3, 95\% CI, 0.2-8.9) (Fig. 12) [18]. The results from the subgroup analyses (2000-2017) for fetal outcomes were consistent with the present findings except for neonatal mortality which was slighly lower in the subgroup analysis (2.9\% vs. 3.8\%) (Additional file 2 ).

\section{Graft outcomes}

The overall acute rejection rate during pregnancy among 822 kidney transplant recipients was 9.4\% (95\% CI, 6.4-13.7), which was comparable to the US mean of 9.1\%. [19] Rates of acute renal allograft rejection were highest in Asia (11.0\%), followed by South America (10.7\%), Oceania (9.1\%), Europe (7.3\%), North America (6.7\%), and Africa (4.8\%) (Fig. 13). With regards to graft failure, there was large variability in the follow up period ranging from 1 year to 14 years. However among 489 recipients in 12 studies where two-year post pregnancy graft loss was reported, there were 32 cases of graft loss $(9.2 \%)$. The change in preconception creatinine and post-pregnancy creatinine, was statistically significant $(1.23 \pm 0.16 \mathrm{mg} / \mathrm{dl}$ vs. $1.37 \pm 0.27 \mathrm{mg} / \mathrm{dl}, p=0.007)$.

\section{Time of conception}

Outcomes were also stratified by interval of $<2$ years, $2-3$ years, and $>3$ years between pregnancy and kidney transplant (Table 2). Adverse pregnancy outcomes of 


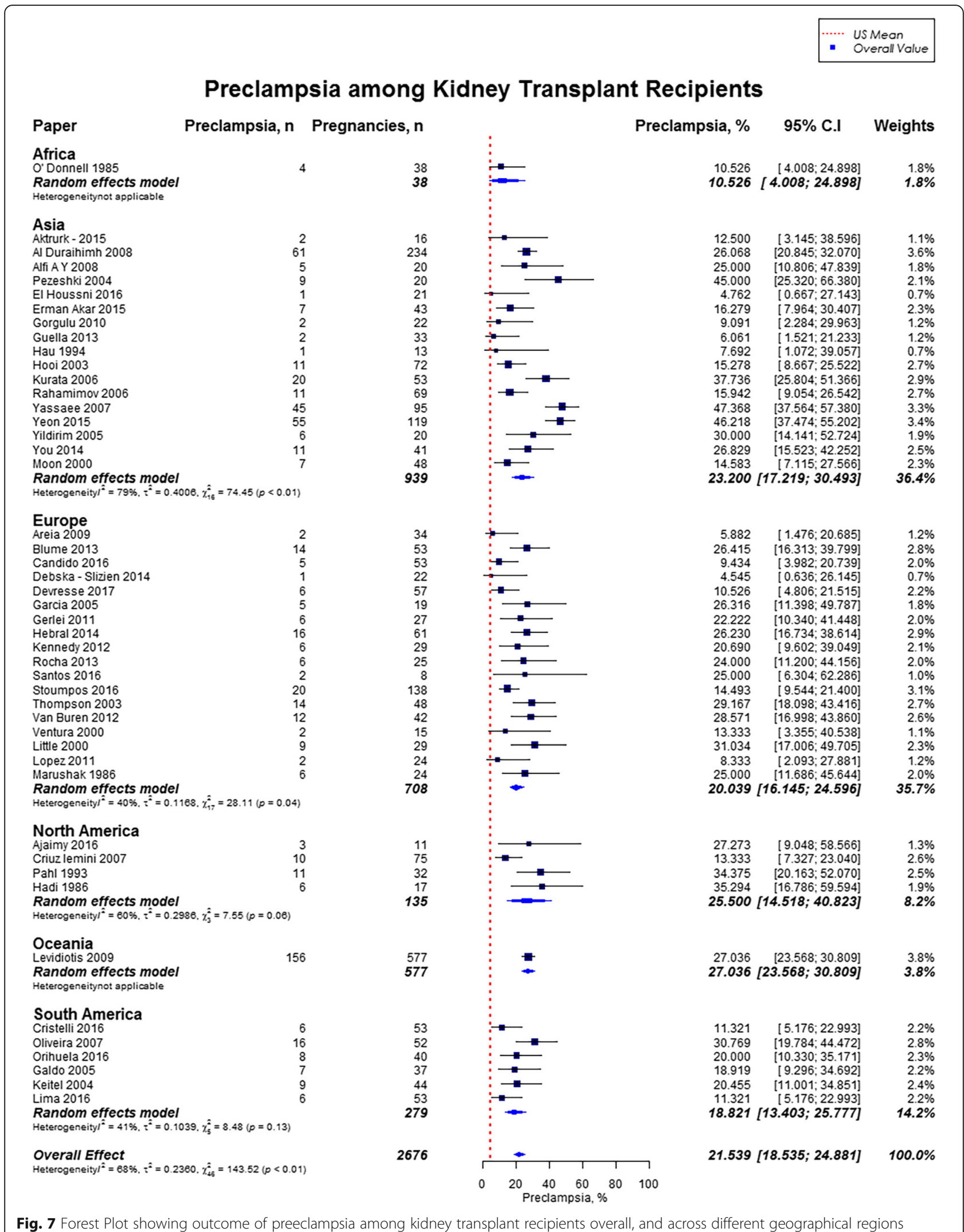




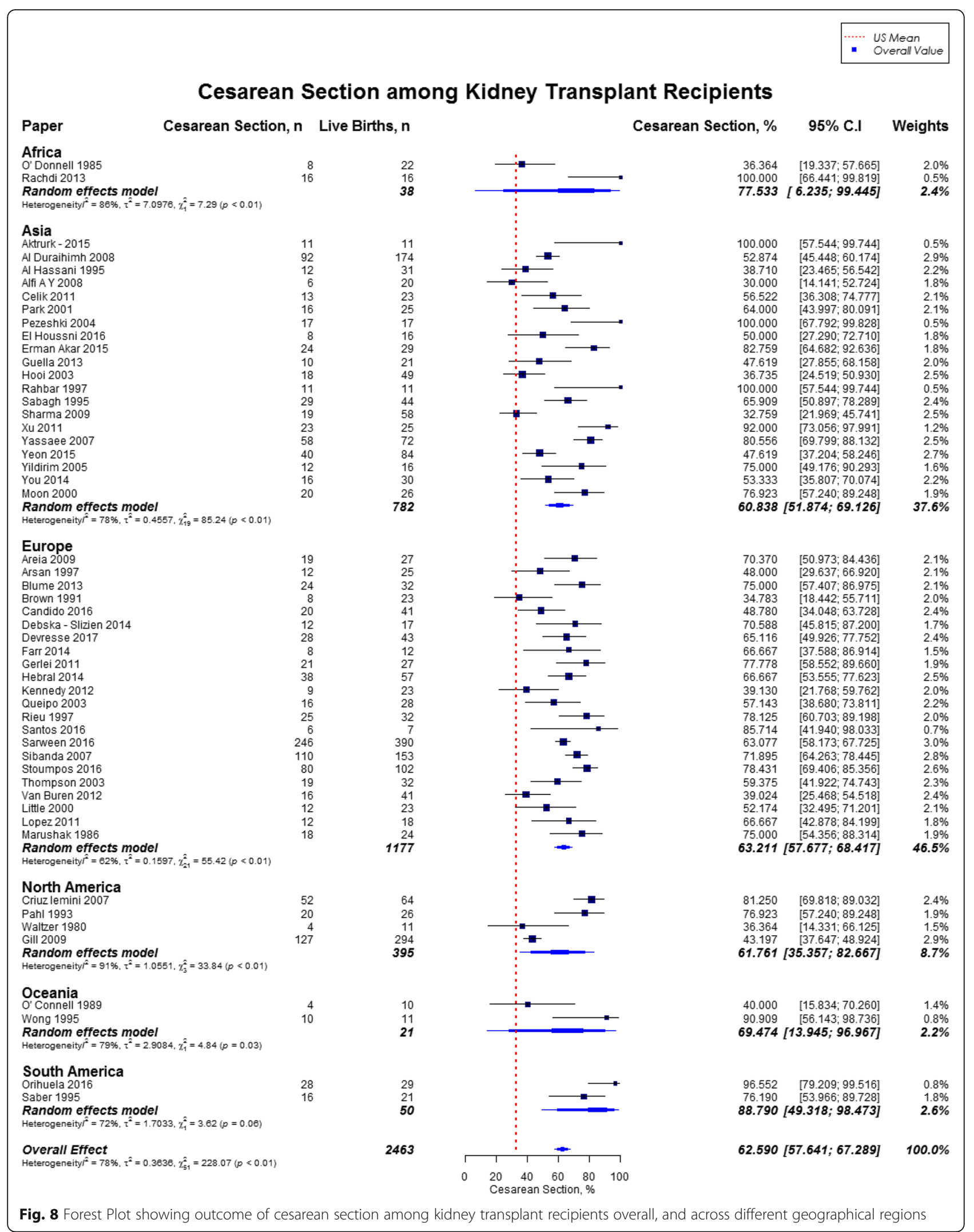




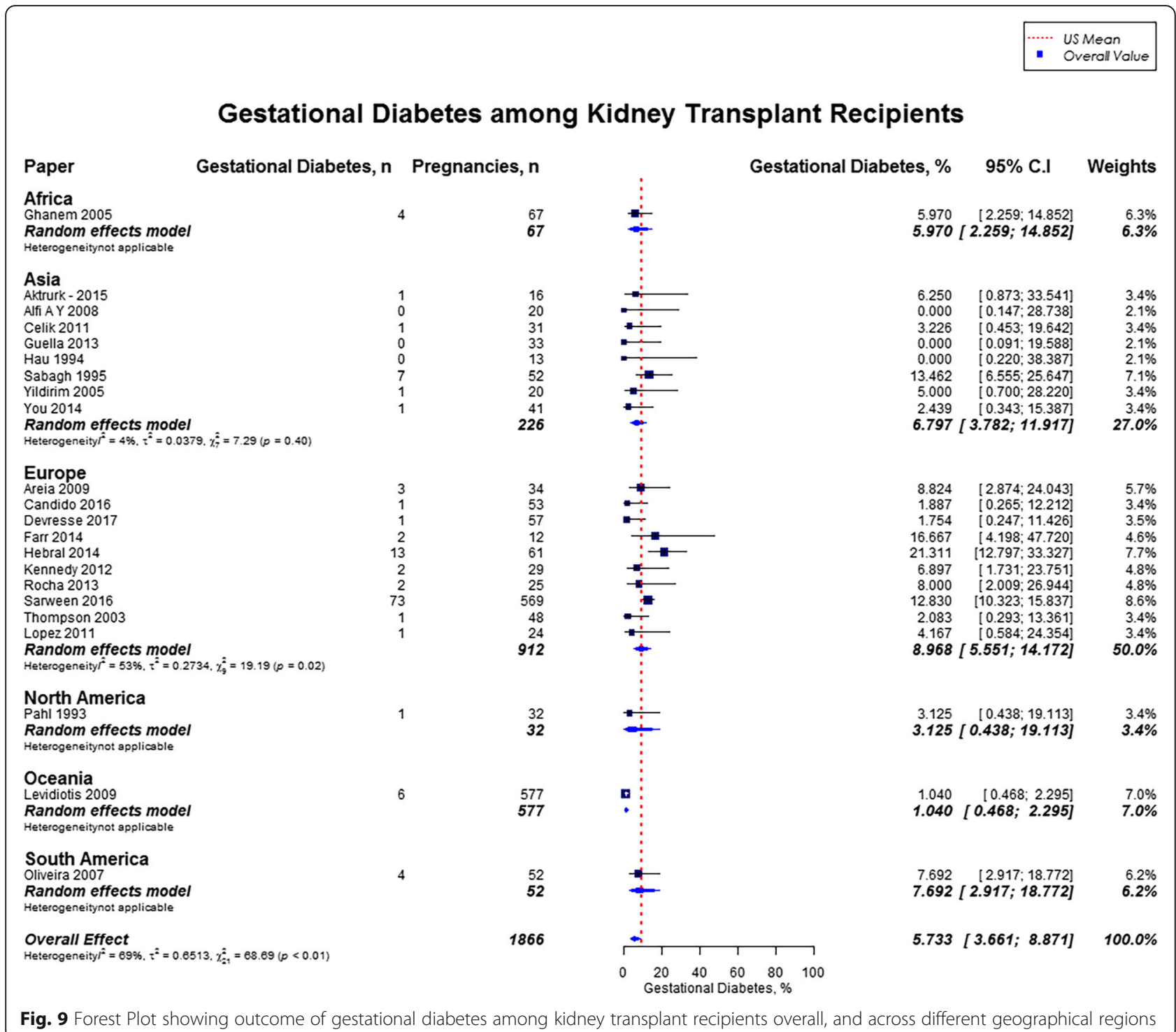

induced abortion rates and neonatal deaths were highest in the 2-3 year interval following kidney transplant as compared to < 2 year interval and $>3$ year interval $(16 \%$ vs. $11 \%$ vs. 10 , and $9 \%$ vs. $3 \%$ vs. $4 \%$ respectively). Cesarean section rate and live birth rate were also less favorable in this interval of $2-3$ years than $>3$ year, and < 2 year interval ( $68 \%$ vs. $75 \%$ vs. 74 , and $73 \%$ vs. $65 \%$ vs. $42 \%$ respectively). Maternal complication of preeclampsia was higher in the $2-3$ interval, and $>3$ year interval than <2year interval ( $24 \%$ vs. $23 \%$ vs. $13 \%)$. Spontaneous abortion rates were highest in $>3$ year interval followed by $2-3$ interval, and $<2$ interval (16\% vs. $14 \%$ vs. $10 \%)$.

\section{Maternal age for conception}

We further stratified the pregnancy, maternal, fetal, and graft outcomes by maternal age categories (Table 3 ).
Lower live birth rate was observed in women with maternal age $29-34$ years than those $<29$ years $(74 \%$ vs. $76 \%$ ). Rates of spontaneous abortion were highest in women $<25$ years and $>35$ years followed by women with maternal age $25-34$ years ( $20 \%$ vs. $18 \%$ vs. $11 \%$ ). Preeclampsia rates were higher in women with maternal age $>35$ years $(27 \%)$ and $29-34$ years $(26 \%)$ followed by $<25$ years $(17 \%)$ and $25-29$ years $(14 \%)$.

\section{Discussion}

The results of our meta-analysis show that although majority of pregnancies in women after kidney transplant result in live birth, both maternal and fetal adverse events are common. Rates of preeclampsia, still birth, and cesarean section were significantly higher than in the general population. In the cohort considered for the analysis, a quarter of women had serious pregnancy 


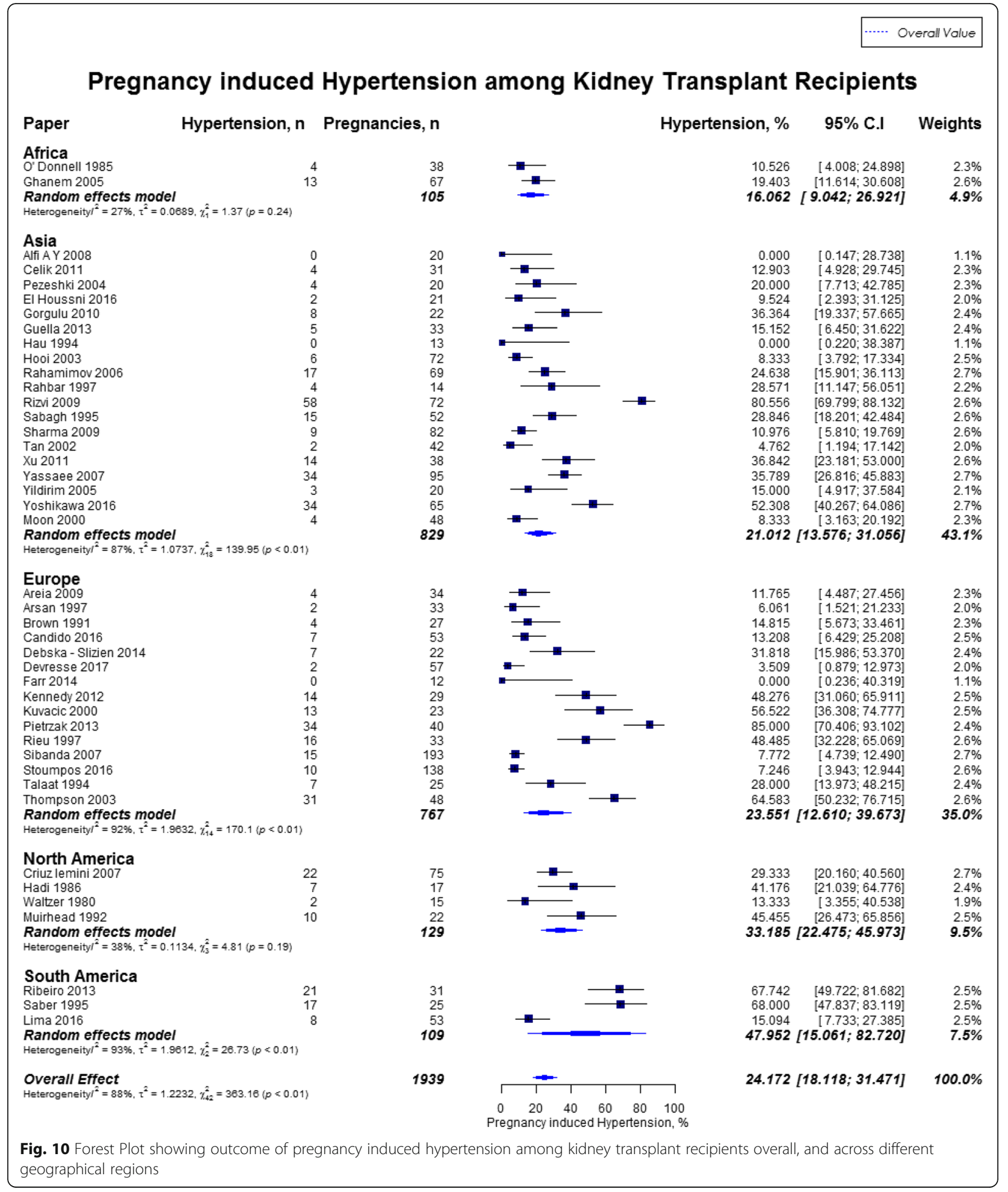

complications, defined as at least one of preterm delivery, first or second trimester loss, stillbirth, or neonatal death. Additionally, rates of preterm delivery, still births, and neonatal mortality were higher as compared with the US recent national data.
The live birth rates in women after kidney transplant were higher than in the general population $(73 \%$ vs. $62 \%)$ and this trend was consistent throughout the globe [10]. Our study confirms the findings from The National Transplant Pregnancy Registry from the United States 


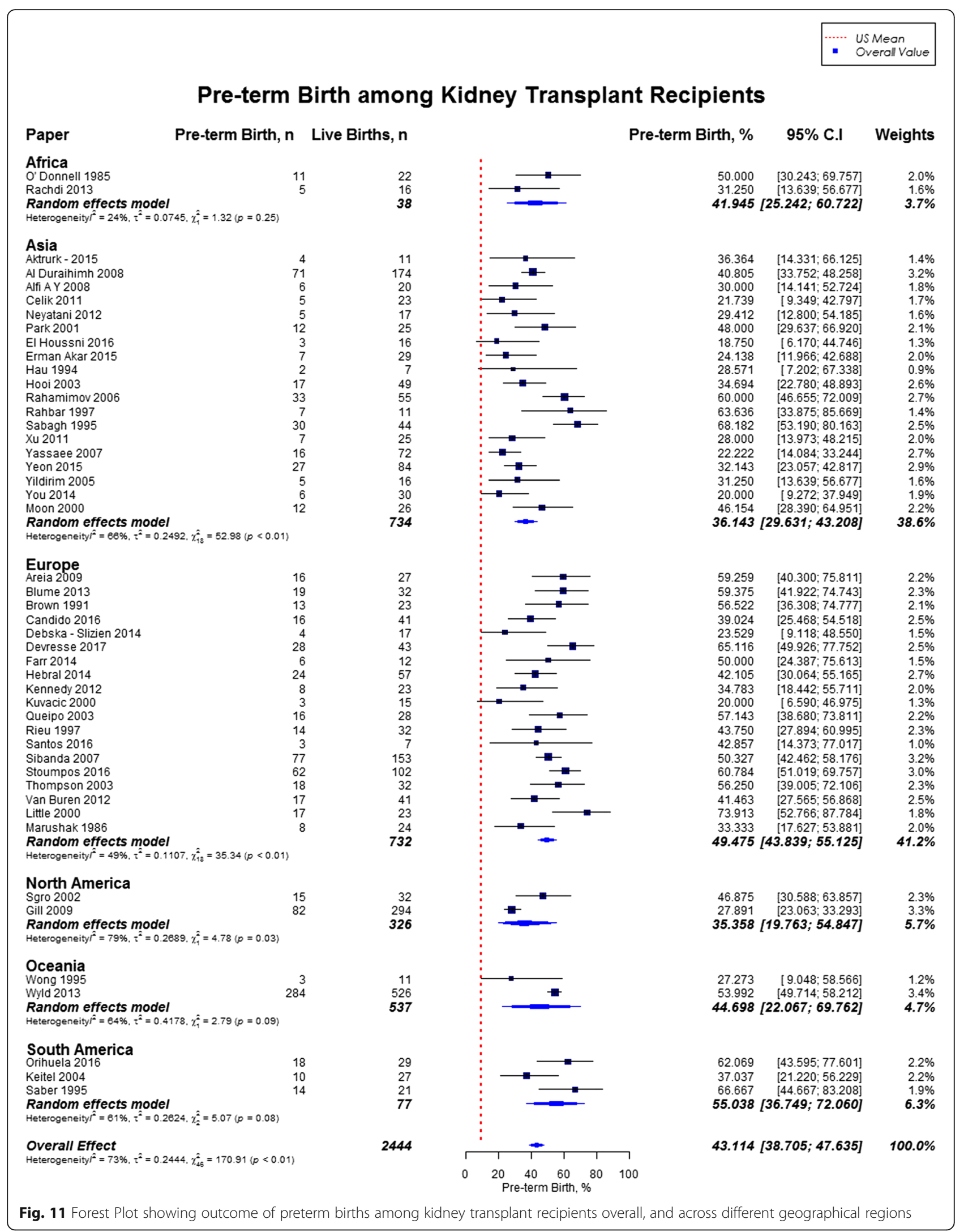




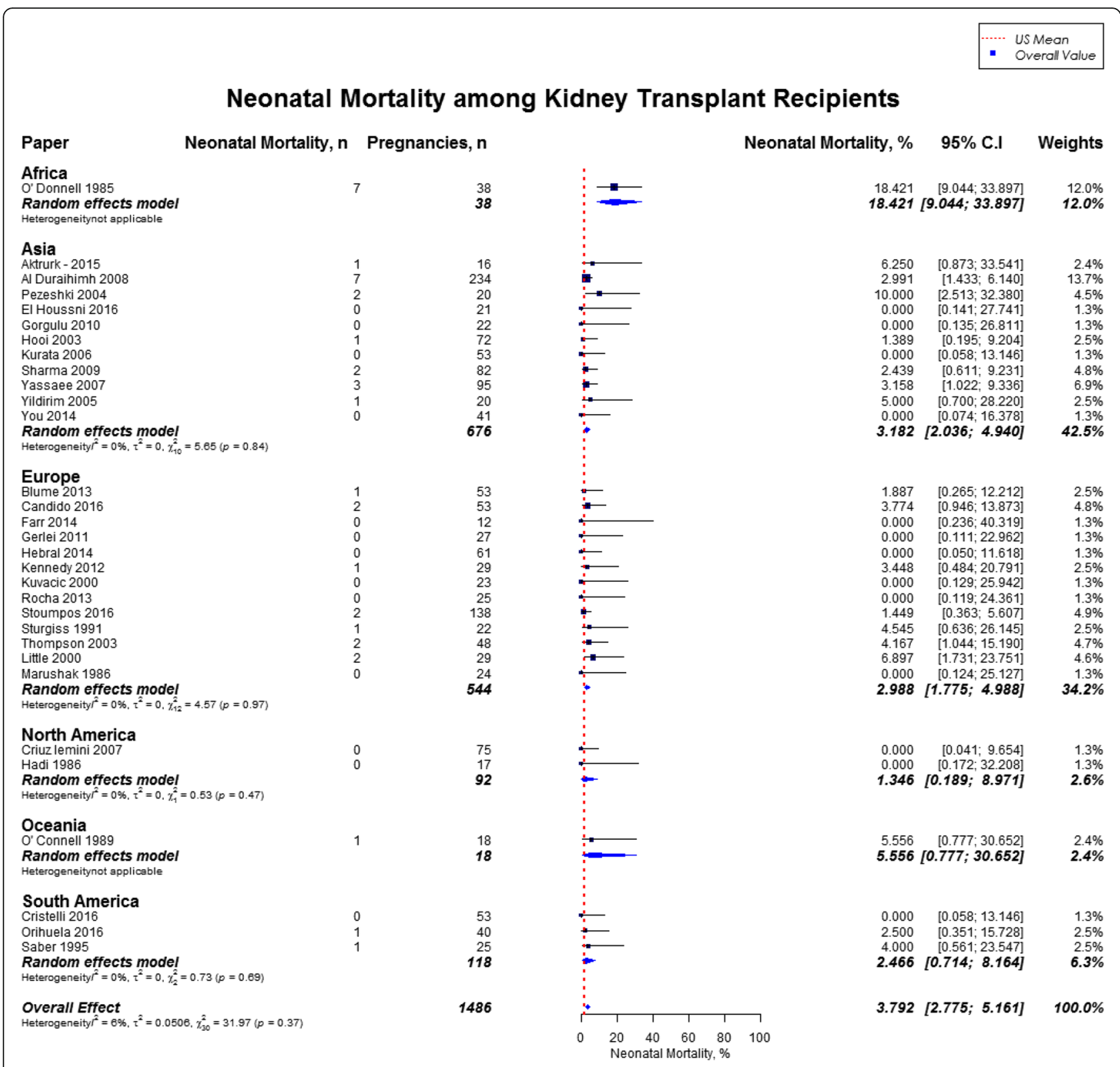

Fig. 12 Forest Plot showing outcome of neonatal mortality among kidney transplant recipients overall, and across different geographical regions

that reported a live birth rate of $71-76 \%$ [7]. Similarly, meta-analysis done by Deshpande et al. examined pregnancy outcomes of 4706 pregnancies in women with kidney transplant and reported a live birth rate of $73.5 \%$ [9]. The higher live birth rate, although appears encouraging, may reflect a reporting bias or a selection bias in which relatively healthy women decided to pursue pregnancy, and subsequently received better medical support by multiple specialties. It is also important to consider that there are inconsistencies in definition of live birth rate used in various studies, for example live birth rate was defined as per 1000 female transplant recipients in some studies, whereas per 1000 pregnancies in transplant recipients in others $[7,20]$. Live birth rate in general population (comparison group) is defined by Centers for Disease Control as live births per 1000 population [10]. Additionally, it remains unclear how the multiple gestation pregnancy outcomes were evaluated in these studies. Contrary to the above findings of successful pregnancies, a US health utilization study found a much lower live birth rate of $55 \%$ in kidney transplant recipients. They attributed this finding of low live birth rate to underestimation of fetal loss [20]. Davison et al. estimated that just under $40 \%$ of conceptions do not go 


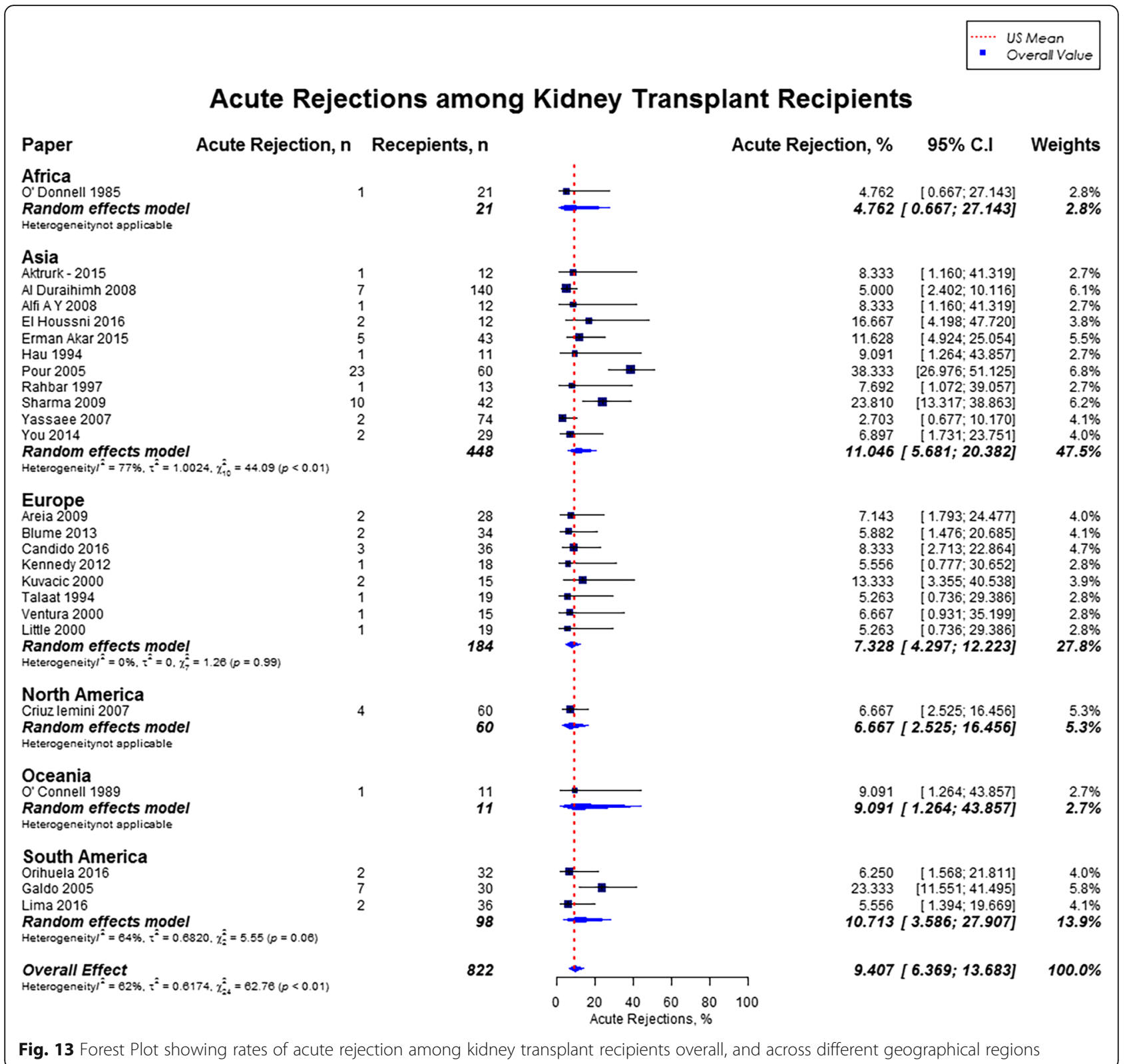

beyond the first trimester, but of those that do, greater than $90 \%$ end successfully [21]. Another explanation of high live birth rate in our study could be the exclusion of studies that reported pregnancy outcomes with teratogenic immunosuppressive medications of mycophenolate and sirolimus.

Our study highlights the significantly higher risk of maternal and fetal complications in women with kidney transplants. About a quarter of women developed preeclampsia, and the rates of preeclampsia were almost six fold higher as compared to the general US population (21.5\% vs. 3.8\%) [16]. Vannevel et al. in an international multicenter retrospective cohort of 52 women who underwent kidney transplantation reported preeclampsia rate of as high as $38 \%$, and chronic hypertension rate of $27 \%$ [22]. Hypertension is common in kidney transplant recipients prior to conception with a reported incidence of 52 to $69 \%$ [1]. Several factors can contribute to the onset of hypertension after renal transplantation, including but not limited to the type of immunosuppressive therapy (calcineurin inhibitors and corticosteroids), allograft function, donor type, obesity, alcohol, smoking, and presence of a native kidney (increased production of renin) [23]. Diagnosis of superimposed preeclampsia can be difficult in kidney transplant patients due to higher frequency of preexisting hypertension and proteinuria [1,24].

We found significant differences in rates of gestational diabetes mellitus between various geographical location, 
Table 2 Pregnancy-related outcomes stratified by study mean interval between transplant and pregnancy

\begin{tabular}{llll}
\hline & $<2$ years & $>2-3$ years & $>3$ years \\
\hline Number of papers & 4 & 15 & 44 \\
Number of pregnancies & 149 & 835 & 3182 \\
Mean maternal age (year) & 28.3 & 29.4 & 29.1 \\
Pregnancy Outcomes & & & \\
$\quad$ Live birth & $73.8 \%$ & $68.3 \%$ & $75.4 \%$ \\
Induced abortion & $10.7 \%$ & $16.1 \%$ & $10.2 \%$ \\
Spontaneous abortion & $10.3 \%$ & $14.0 \%$ & $16.3 \%$ \\
Still birth & $6.7 \%$ & $5.1 \%$ & $3.7 \%$ \\
$\quad$ Neonatal deaths & $3.4 \%$ & $9.3 \%$ & $3.7 \%$ \\
Cesarean section & $41.8 \%$ & $72.7 \%$ & $64.5 \%$ \\
Maternal Outcomes & & & \\
Preeclampsia & $13.2 \%$ & $24.3 \%$ & $22.8 \%$ \\
Pregnancy induced hypertension & $12.1 \%$ & $30.8 \%$ & $23.0 \%$ \\
Gestational diabetes & $0.0 \%$ & $8.8 \%$ & $7.2 \%$ \\
Fetal Outcomes & & & \\
Pre-term delivery & $41.9 \%$ & $41.6 \%$ & $45.4 \%$ \\
Mean gestation time (weeks) & 36.1 & 34.5 & 34.9 \\
Birth weight (grams) & 2349.00 & 2533.21 & 2460.79 \\
Graft Outcomes & & & \\
Acute rejection & $8.1 \%$ & $5.1 \%$ & $3.0 \%$ \\
Graft loss & $16.7 \%$ & $14.6 \%$ & $6.3 \%$ \\
\hline
\end{tabular}

for example rates were as high as $8.9 \%$ in Europe and as low as $1 \%$ in Oceania. Although, the increased rate of gestational diabetes in kidney transplant patients can be well explained by the use of immunosuppressive medications like steroids and calcineurin inhibitors, the striking differences between rates of gestational diabetes according to geographic location also highlights the importance of predisposition to diabetes due to ethnicity. Unfortunately, it was not possible to evaluate the differences in immunosuppressive medications as usually they are individualized to the needs of the patients and transplant center protocol $[1,25]$.

Rates of stillbirth and neonatal mortality were significantly higher in our study as compared to the general population. While prior studies have not reported higher rates of neonatal mortality and stillbirths in kidney transplant recipients, the current study finding is highly significant. Possible reasons could be prematurity, preeclampsia or presence of other risk factors like hypertension, proteinuria, and serum creatinine of $1.5 \mathrm{mg} / \mathrm{dl}$ or higher [26-28]. While it was not possible to determine the exact cause for stillbirth or neonatal mortality, this study finding is critical for counselling of women of child bearing age contemplating pregnancy. In our study, the rate of cesarean section was higher than two folds as
Table 3 Pregnancy-related outcomes stratified by study mean maternal age

\begin{tabular}{|c|c|c|c|c|}
\hline \multicolumn{5}{|l|}{ Study mean maternal age (years) } \\
\hline & $<25$ & $25-29$ & $30-34$ & $\geq 35$ \\
\hline Number of papers & 3 & 22 & 35 & 1 \\
\hline Number of pregnancies & 103 & 723 & 3474 & 11 \\
\hline Mean maternal age (year) & 23.3 & 27.4 & 30.2 & 36.0 \\
\hline \multicolumn{5}{|l|}{ Pregnancy Outcomes } \\
\hline Live birth & $75.8 \%$ & $75.8 \%$ & $73.9 \%$ & na \\
\hline Induced abortion & $14.0 \%$ & $11.3 \%$ & $11.0 \%$ & na \\
\hline Spontaneous abortion & $19.8 \%$ & $16.0 \%$ & $13.3 \%$ & $18.2 \%$ \\
\hline Still birth & $2.9 \%$ & $5.3 \%$ & $3.6 \%$ & $9.1 \%$ \\
\hline Neonatal deaths & na & $5.4 \%$ & $3.0 \%$ & na \\
\hline Cesarean section & $48.0 \%$ & $68.3 \%$ & $63.6 \%$ & na \\
\hline \multicolumn{5}{|l|}{ Maternal Outcomes } \\
\hline Preeclampsia & $17.1 \%$ & $13.7 \%$ & $26.5 \%$ & $27.3 \%$ \\
\hline Pregnancy induced hypertension & $16.5 \%$ & $25.2 \%$ & $23.4 \%$ & na \\
\hline Gestational diabetes & na & $5.8 \%$ & $7.0 \%$ & na \\
\hline \multicolumn{5}{|l|}{ Fetal Outcomes } \\
\hline Pre-term delivery & na & $46.4 \%$ & $47.4 \%$ & na \\
\hline Mean gestation time (weeks) & 35.5 & 35.5 & 34.6 & na \\
\hline Birth weight (grams) & 2460.0 & 2607.7 & 2456.9 & na \\
\hline \multicolumn{5}{|l|}{ Graft Outcomes } \\
\hline Acute rejection & $3.8 \%$ & $3.3 \%$ & $5.8 \%$ & na \\
\hline Graft loss & na & $12.1 \%$ & $10.4 \%$ & $27.3 \%$ \\
\hline
\end{tabular}

${ }^{*}$ na not available

compared to general population in United States, and varied from 60 to $77 \%$ across different geographical locations. Bramham et al. reported that more than three quarters of the deliveries in kidney transplant recipients were by cesarean section, but only $3 \%$ were performed for the indication of renal transplant [3]. Vaginal delivery should not be impaired in kidney transplant patients, as the pelvic allograft does not obstruct the birth canal in most patients [1]. This exceptionally higher rates of cesarean sections in kidney transplant recipients can be attributed to fetal and maternal complications, but warrants further study. There was a high rate of premature births in the transplant population in the present study and close to half of the live births were premature deliveries. Prior studies have showed a preterm birth rate of $40-60 \%$ in kidney transplant recipients [9, 29]. Fetal complications, suspected renal compromise or preeclampsia are some of the common indications of early iatrogenic delivery. Interestingly, only quarter of preterm deliveries in renal transplant recipients are induced [3, 7].

The optimal time to conception after renal transplant continues to remain an area of contention. The ideal 
time of conception in women with renal transplant is between 1 and 2 years after transplantation according to guidelines by American Society of Transplantation, whereas European best practice guidelines recommend delaying pregnancy for a period of 2 years after transplantation [30, 31]. In our study, live birth rate was lowest and neonatal deaths were highest in the 2-3 year interval following kidney transplant. Maternal complication of cesarean section and preeclampsia were higher in the $2-3$ and $>3$ year interval. In contrast, Deshpande et al. reported both the highest maternal complications of preeclampsia, cesarean section, and gestational diabetes, and least favorable delivery outcome of preterm births in the $<2$ year interval as compared to $>2$ year interval between kidney transplant and pregnancy [9]. However their analysis was limited by inclusion of only 3 studies in the $<2$ interval following kidney transplant. Overall, fetal outcomes in $<2$ year interval seem most favorable in our study but merits further investigation due to limitation of the retrospective study design, small numbers, and possible reporting bias associated with data from voluntary registries.

A significant strength of our study is that it involves a large number of pregnant renal transplant recepients from all around the globe, thus providing us with information about pregnancy outcomes for a heterogenous population. Additionally, we have analyzed region specific outcomes and identified outcomes which may require intensive management pertaining to that region. This will help in making future region specific guidelines for follow up and management of pregnancy in kidney transplant recpients. The following limitations should be considered when interpreting the findings of our study. We examined pregnancy outcomes over several decades in the present study. While it is expected for the outcomes to change due to improvement in obstetric care in kidney transplant recipients over the course of time, subgroup analysis for studies from 2000 to 2017 showed consistent results. There were inconsistencies in the definition of live birth rate amongst different studies that may have affected the results. Reporting bias may have affected the miscarriage rate. We were unable to account for differences in socioeconomics, and healthcare conditions among the different geographic regions. Due to lack of individual patient data, we were not able to assess pregnancy outcomes in relation to immunosuppression regimens.

\section{Conclusions}

This meta analysis of pregnancy outcomes in 6712 pregnancies in 4174 kidney transplant recipients with data spread over different decades from all over the world shows favorable outcomes with live birth rates exceeding that in the recent national population. Majority of patients preserve their graft. However, pregnancy after renal transplant confers significant risk in terms of maternal and fetal adverse events, including increased rates of preeclampsia, gestational diabetes, cesarean section rates, and pregnancy induced hypertension. The risk of prematurity and low birth rate are also high. Areas which need to be studied in the future include type of immunosuppression and its correlation with specific pregnancy outcomes; and evaluation of risk factors associated with specific maternal and fetal adverse events. The definitions used in evaluating these outcomes also need to be standardized. The results of this study can help the health care providers with appropriate counseling and individualized management of this high risk population.

\section{Additional files}

Additional file 1: Reproducible search strategy. (DOCX 149 kb)

Additional file 2: Subgroup analysis of various pregnancy outcomes in kidney transplant recipients for studies published from 2000 to 2017. (DOCX $16 \mathrm{~kb}$ )

\section{Abbreviations}

Cl: Confidence interval; US: United States

\section{Acknowledgements}

None.

\section{Author contributions}

SS initiated the study, designed the study and wrote the initial manuscript. RV, Ayank Gupta, RJ and MS contributed to the study design, and study figures, analyzed and interpreted the data, and did the manuscript review. JW contributed to the sudy design, data analysis, interpretation of data, and manuscript review. EK contibuted to literature search, and manuscript review. TK contributed in study design and manuscript review. Anu Gupta contributed in manuscript writing and manuscript review. TG contributed to the study design, implementation of the study, study figures, and manuscript review. PV assisted SS with study design and implementation, revision of the manuscript and did the final approval of the manuscript. All authors reviewed the manuscript.

\section{Funding}

This work was supported by grant awarded to SS from University of Cincinnati's College of Medicine-Health Sciences Library.

\section{Availability of data and materials}

The datasets used and/or analysed during the current study are available from the corresponding author on reasonable request.

\section{Ethics approval and consent to participate \\ Not applicable.}

\section{Consent for publication}

Not applicable.

\section{Competing interests}

All the authors have no disclosures and competing interests. The results presented in this paper have not been published previously in whole or part, except in abstract format.

\section{Publisher's Note}

Springer Nature remains neutral with regard to jurisdictional claims in published maps and institutional affiliations. 


\section{Author details}

'Division of Nephrology Kidney C.A.R.E. Program, University of Cincinnati, 231 Albert Sabin Way, MSB 6112, Cincinnati, OH 45267, USA. ${ }^{2}$ Department of Environmental Health, University of Cincinnati, Cincinnati, OH, USA. ${ }^{3}$ Health Sciences Library, College of Medicine, University of Cincinnati, Cincinnati, $\mathrm{OH}$, USA. ${ }^{4}$ Buffalo Medical Group, Buffalo, NY, USA. ${ }^{5}$ Division of Neonatology, Cincinnati Children's Hospital and Medical Center, Cincinnati, OH, USA.

Received: 30 September 2018 Accepted: 15 January 2019

Published online: 23 January 2019

\section{References}

1. Shah S, Verma P. Overview of pregnancy in renal transplant patients. International journal of nephrology. 2016;2016:4539342.

2. Saha MT, Saha HH, Niskanen LK, Salmela KT, Pasternack Al. Time course of serum prolactin and sex hormones following successful renal transplantation. Nephron. 2002;92(3):735-7.

3. Bramham K, Nelson-Piercy C, Gao H, Pierce M, Bush N, Spark P, Brocklehurst $P$, Kurinczuk JJ, Knight M. Pregnancy in renal transplant recipients: a UK national cohort study. Clin J Am Soc Nephrol : CJASN. 2013;8(2):290-8.

4. Sifontis NM, Coscia LA, Constantinescu S, Lavelanet AF, Moritz MJ, Armenti VT. Pregnancy outcomes in solid organ transplant recipients with exposure to mycophenolate mofetil or sirolimus. Transplantation. 2006;82(12):1698-702

5. Davison JM, Redman CW. Pregnancy post-transplant: the establishment of a UK registry. Br J Obstet Gynaecol. 1997;104(10):1106-7.

6. Rizzoni $\mathrm{G}$, Ehrich $\mathrm{JH}$, Broyer $M$, Brunner FP, Brynger $H$, Fassbinder W Geerlings W, Selwood NH, Tufveson G, Wing AJ. Successful pregnancies in women on renal replacement therapy: report from the EDTA registry. Nephrol Dial Transplant. 1992;7(4):279-87.

7. Coscia LA, Constantinescu S, Moritz MJ, Frank AM, Ramirez CB, Maley WR, Doria C, McGrory CH, Armenti VT. Report from the National Transplantation Pregnancy Registry (NTPR): outcomes of pregnancy after transplantation. Clin Transpl. 2010:65-85.

8. Levidiotis V, Chang S, McDonald S. Pregnancy and maternal outcomes among kidney transplant recipients. Journal of the American Society of Nephrology : JASN. 2009;20(11):2433-40.

9. Deshpande NA, James NT, Kucirka LM, Boyarsky BJ, Garonzik-Wang JM Montgomery RA, Segev DL. Pregnancy outcomes in kidney transplant recipients: a systematic review and meta-analysis. Am J Transplant. 2011; 11(11):2388-404

10. Martin J. Births in the United States, 2016. NCHS data brief, no 287. In: Hamilton B, editor. . Hyattsville MD: National Center for Health Statistics; 2017

11. Martin J. Births: Final Data for 2016. National vital statistics reports; vol 67 no 1. Hyattsville, MD: National Center for Health Statistics; 2018.

12. Martin J: Measuring Gestational Age in Vital Statistics Data: Transitioning to the Obstetric Estimate. National vital statistics reports; vol 64 no 5. Hyattsville, MD: National Center for Health Statistics. 2015. In.

13. Jatlaoui T: Abortion surveillance - United States, 2014. MMWR Surveill Summ 2017. In., vol. 66; 2017: 1-48

14. MacDorman M. Fetal and Perinatal Mortality: United States, 2013. National Vital Statistics Reports; vol 64 no 8. Hyattsville, MD: National Center for Health Statistics 2015; 2015

15. Stulberg DB, Cain LR, Dahlquist I, Lauderdale DS. Ectopic pregnancy rates and racial disparities in the Medicaid population, 2004-2008. Fertil Steril. 2014;102(6):1671-6.

16. Ananth CV, Keyes KM, Wapner RJ. Pre-eclampsia rates in the United States, 1980-2010: age-period-cohort analysis. BMJ (Clinical research ed). 2013:f6564:347.

17. Donahue SM, Kleinman KP, Gillman MW, Oken E. Trends in birth weight and gestational length among singleton term births in the United States: 19902005. Obstet Gynecol. 2010;115(2 Pt 1):357-64.

18. Broder A. Pregnancy outcomes in renal transplants with and without lupus. Clinical and Translational Science. 2013;6(2):128.

19. Tanriover B, Jaikaransingh $V$, MacConmara MP, Parekh JR, Levea SL, Ariyamuthu VK, Zhang S, Gao A, Ayvaci MU, Sandikci B, et al. Acute rejection rates and graft outcomes according to induction regimen among recipients of kidneys from deceased donors treated with tacrolimus and mycophenolate. Clin J Am Soc Nephrol. 2016;11(9):1650-61.

20. Gill JS, Zalunardo N, Rose C, Tonelli M. The pregnancy rate and live birth rate in kidney transplant recipients. Am J Transplant. 2009;9(7):1541-9.
21. Davison JM. Renal transplantation and pregnancy. American journal of kidney diseases : the official journal of the National Kidney Foundation. 1987;9(4):374-80.

22. Vannevel V, Claes K, Baud D, Vial Y, Golshayan D, Yoon EW, Hodges R, Le Nepveu A, Kerr PG, Kennedy C, et al. Preeclampsia and long-term renal function in women who underwent kidney transplantation. Obstet Gynecol. 2018;131(1):57-62

23. Haas M, Mayer G. Cyclosporin A-associated hypertension--pathomechanisms and clinical consequences. Nephrol Dial Transplant. 1997;12(3):395-8.

24. Aivazoglou L, Sass N, Silva HT Jr, Sato JL, Medina-Pestana JO, De Oliveira LG. Pregnancy after renal transplantation: an evaluation of the graft function. Eur J Obstet Gynecol Reprod Biol. 2011;155(2):129-31.

25. McKay DB, Josephson MA. Pregnancy after kidney transplantation. Clin J Am Soc Nephrol : CJASN. 2008;3(Suppl 2):S117-25.

26. Majak GB, Reisaeter AV, Zucknick M, Lorentzen B, Vangen S, Henriksen T, Michelsen TM. Preeclampsia in kidney transplanted women; outcomes and a simple prognostic risk score system. PLoS One. 2017;12(3):e0173420.

27. Little MA, Abraham KA, Kavanagh J, Connolly G, Byrne P, Walshe JJ. Pregnancy in Irish renal transplant recipients in the cyclosporine era. Ir J Med Sci. 2000;169(1):19-21.

28. Cruz Lemini MC, Ibarguengoitia Ochoa F, Villanueva Gonzalez MA. Perinatal outcome following renal transplantation. Int J Gynaecol Obstet. 2007;96(2):76-9.

29. Mohammadi FA, Borg M, Gulyani A, McDonald SP, Jesudason S. Pregnancy outcomes and impact of pregnancy on graft function in women after kidney transplantation. Clin Transpl. 2017:31(10).

30. European best practice guidelines for renal transplantation. Section IV: Long-term management of the transplant recipient. IV.10. Pregnancy in renal transplant recipients. Nephrol Dial Transplant. 2002;17(Suppl 4):50-5.

31. McKay DB, Josephson MA, Armenti VT, August P, Coscia LA, Davis CL, Davison JM, Easterling T, Friedman JE, Hou S, et al. Reproduction and transplantation: report on the AST consensus conference on reproductive issues and transplantation. Am J Transplant. 2005;5(7):1592-9.

32. Devresse A, Jassogne C, Hubinont C, De Meyer M, Mourad M, Goffin E, Kanaan N. Maternal risks and pregnancy outcomes after kidney transplantation: a single center experience. Am J Transplant. 2017;17:253.

33. Yuksel Y, Tekin S, Yuksel D, Duman I, Sarier M, Yucetin L, Turan E, Celep H, Ugurlu T, Inal MM, et al. Pregnancy and delivery in the sequel of kidney transplantation: single-center study of 8 Years' experience. Transplant Proc. 2017;49(3):546-50

34. Ajaimy M, Lubetzky M, Jones T, Kamal L, Colovai A, de Boccardo G, Akalin E Pregnancy in sensitized kidney transplant recipients: a single-center experience. Clin Transpl. 2016;30(7):791-5.

35. Candido C, Cristelli MP, Fernandes AR, Lima AC, Viana LA, Sato JL, Sass N, Tedesco-Silva H, Medina-Pestana JO. Pregnancy after kidney transplantation: high rates of maternal complications. J Bras Nefrol. 2016;38(4):421-6.

36. Cristelli M, Candido CD, Fernandes AR, Viana LA, Sato JL, Sass N, De Lima ACA, Tedesco-Silva H, Medina-Pestana JO. Pregnancy among kidney transplanted women: feasibility of pregnancy and effects on the mother and renal graft. Pregnancy Hypertension. 2016;6(3):232.

37. El Houssni S, Sabri S, Benamar L, Ouzeddoun N, Bayahia R, Rhou H. Pregnancy after renal transplantation: effects on mother, child, and renal graft function. Saudi J Kidney Dis Transpl. 2016;27(2):227-32.

38. Lima A, Cristelli M, Teixeira C, Pietrobom I, Basso G, Viana L, De Paula M, Cândido C, Tedesco-Silva H, Pestana J. Pregnancy in the renal transplant recipient: pregnancy viability and effects on graft function. Am J Transplant. 2016;16:783-4.

39. Majak GB, Sandven I, Lorentzen B, Vangen S, Reisaeter AV, Henriksen T, Michelsen TM. Pregnancy outcomes following maternal kidney transplantation: a national cohort study. Acta Obstet Gynecol Scand. 2016;95(10):1153-61.

40. Mishra W, Nanda S, Mistry K, Aggarwal R, Choudhary S, Gandhi K. Pregnancy outcome in renal transplant recipients: Indian scenario. Indian Journal of Transplantation. 2016;10(3):57-60.

41. Orihuela S, Nin M, San Roman S, Noboa O, Curi L, Silvarino R, GonzalezMartinez F. Successful pregnancies in kidney transplant recipients: experience of the National Kidney Transplant Program from Uruguay. Transplant Proc. 2016:48(2):643-5.

42. Piccoli GB, Cabiddu G, Attini R, Gerbino M, Todeschini P, Perrino ML, Manzione AM, Piredda GB, Gnappi E, Caputo F, et al. Pregnancy outcomes after kidney graft in Italy: are the changes over time the result of different therapies or of different policies? A nationwide survey (1978-2013). Nephrol Dial Transplant. 2016;31(11):1957-65. 
43. Saliem S, Patenaude V, Abenhaim HA. Pregnancy outcomes among renal transplant recipients and patients with end-stage renal disease on dialysis. J Perinat Med. 2016:44(3):321-7.

44. Santos S, Malheiro J, Campos A, Pedroso S, Almeida M, Martins LS, Dias L, Henriques C, Cabrita A. Pregnancy in renal transplant recipients: obstetric outcomes and risk of allosensitization. Medicine. 2016;95(10).

45. Sarween N, Hughes S, Evison F, Day C, Knox E, Lipkin G. Pregnancy outcomes in renal transplant recipients in England over 15 years. Nephrology Dialysis Transplantation. 2016;31:i6.

46. Stoumpos S, MCNeill SH, Gorrie M, Mark PB, Brennand JE, Geddes CC, Deighan CJ. Obstetric and long-term kidney outcomes in renal transplant recipients: a 40-yr single-center study. Clin Transpl. 2016;30(6):673-81.

47. Yoshikawa Y, Uchida J, Akazawa C, Suganuma N. Analyses of relationship between obstetric complications and preterm delivery in Japanese recipients received kidney transplant. Transplantation. 2016;100(7):S884-5.

48. Akturk S, Celebi ZK, Erdogmus S, Kanmaz AG, Yuce T, Sengul S, Keven K. Pregnancy after kidney transplantation: outcomes, tacrolimus doses, and trough levels. Transplant Proc. 2015;47(5):1442-4.

49. Arab K, Oddy L, Patenaude V, Abenhaim HA. Obstetrical and neonatal outcomes in renal transplant recipients. J Matern Fetal Neonatal Med. 2015;28(2):162-7.

50. Erman Akar M, Ozekinci M, Sanhal C, Kececioglu N, Mendilcioglu I, Senol Y, Dirican K, Kocak H, Dinckan A, Suleymanlar G. A retrospective analysis of pregnancy outcomes after kidney transplantation in a single center. Gynecol Obstet Investig. 2015;79(1):13-8.

51. Yeon HK, Shim JY, Won HS, Lee PR, Kim A. Risk factors for developing preeclamptic disorder in the pregnancy after kidney transplantation. J Perinat Med. 2015;43:1293.

52. Debska-Slizien A, Galgowska J, Chamienia A, Bullo-Piontecka B, Krol E, Lichodziejewska-Niemierko M, Lizakowski S, Renke M, Rutkowski P, Zdrojewski Z, et al. Pregnancy after kidney transplantation: a single-center experience and review of the literature. Transplant Proc. 2014;46(8):2668-72.

53. Farr A, Bader $Y$, Husslein PW, Gyori G, Muhlbacher F, Margreiter M. Ultrahigh-risk pregnancies in women after renal transplantation. Eur J Obstet Gynecol Reprod Biol. 2014;180:72-6.

54. Hebral AL, Cointault O, Connan L, Congy-Jolivet N, Esposito L, CardeauDesangles I, Del Bello A, Lavayssiere L, Nogier MB, Ribes D, et al. Pregnancy after kidney transplantation: outcome and anti-human leucocyte antigen alloimmunization risk. Nephrol Dial Transplant. 2014;29(9):1786-93.

55. You JY, Kim MK, Choi SJ, Oh SY, Kim SJ, Kim JH, Oh HY, Roh CR. Predictive factors for adverse pregnancy outcomes after renal transplantation. Clin Transpl. 2014;28(6):699-706.

56. Blume C, Sensoy A, Gross MM, Guenter HH, Haller H, Manns MP, Schwarz A, Lehner F, Klempnauer J, Pischke S, et al. A comparison of the outcome of pregnancies after liver and kidney transplantation. Transplantation. 2013;95(1):222-7.

57. Guella A, Mohamed E. Pregnancy in Saudi kidney transplant recipients: a local experience. Transpl Int. 2013;26:287.

58. Pietrzak B, Songin T, Szymusik I, Najman BK, Jabiry-Zieniewicz Z, Cyganek A, Wielgos A, Wielgos M. Pregnancy in female liver or kidney transplant recipients. J Perinat Med. 2013:41.

59. Rachdi M, Amine BH, Mohamed B, Mounir C, Radhouane R. Pregnancy after renal transplantation and neonatal outcome. J Perinat Med. 2013;41.

60. Ribeiro R, Grecco R, Ribeiro T, Pulcineli R, Francisco V, Pacheco V, Soubhi Z, Zugaib KM. Analysis of obstetrical and neonatal outcomes in pregnant women post renal transplantation. J Perinat Med. 2013;41.

61. Rocha A, Cardoso A, Malheiro J, Martins LS, Fonseca I, Braga J, Henriques AC. Pregnancy after kidney transplantation: graft, mother, and newborn complications. Transplant Proc. 2013;45(3):1088-91.

62. Wyld ML, Clayton PA, Jesudason S, Chadban SJ, Alexander SI. Pregnancy outcomes for kidney transplant recipients. Am J Transplant. 2013;13(12):3173-82.

63. Kennedy C, Hussein W, Spencer S, Walshe J, Denton M, Conlon PJ, Magee C. Reproductive health in Irish female renal transplant recipients. Ir J Med Sci. 2012;181(1):59-63.

64. Neyatani N, Yamaguchi N, Takagi H, Fujii R, Makinoda S. Clinical study on fertility in women received renal transplantation. Int J Gynecol Obstet. 2012; 119:S435-6.

65. Van Buren MC, Van De Wetering J, Roodnat J, Berger SP, Tielen M, Weimar W. Pregnancy after kidney transplantation: 'Will mothers see their children grow up?'. Transplantation. 2012;94:141.

66. Celik G, Toz H, Ertilav M, Asgar N, Ozkahya M, Basci A, Hoscoskun C. Biochemical parameters, renal function, and outcome of pregnancy in kidney transplant recipient. Transplant Proc. 2011;43(7):2579-83.
67. Gerlei Z, Wettstein D, Rigo J, Asztalos L, Langer RM. Childbirth after organ transplantation in Hungary. Transplant Proc. 2011;43(4):1223-4.

68. Lopez V, Martinez D, Vinolo C, Cabello M, Sola E, Gutierrez C, Burgos D, Gonzalez-Molina M, Hernandez D. Pregnancy in kidney transplant recipients: effects on mother and newborn. Transplant Proc. 2011;43(6):2177-8.

69. Xu LG, Han S, Liu Y, Wang HW, Yang YR, Qiu F, Peng WL, Tang LG. Timing, conditions, and complications of post-operative conception and pregnancy in female renal transplant recipients. Cell Biochem Biophys. 2011;61(2):421-6.

70. Gorgulu N, Yelken B, Caliskan Y, Turkmen A, Sever MS. Does pregnancy increase graft loss in female renal allograft recipients? Clin Exp Nephrol. 2010;14(3):244-7.

71. Areia A, Galvao A, Pais MS, Freitas L, Moura P. Outcome of pregnancy in renal allograft recipients. Arch Gynecol Obstet. 2009;279(3):273-7.

72. Rizvi SAH, Kumari K, Naqvi R, Aziz T, Ahmed E, Naqvi SAA. An update on pregnancy outcome in renal transplant recipients. Am J Transplant. 2009;9:518-9.

73. Sharma U, Minocha S. Pregnancy after renal transplant - Oman perspective. Int J Gynecol Obstet. 2009;107:S269.

74. Al Duraihimh H, Ghamdi G, Moussa D, Shaheen F, Mohsen N, Sharma U, Stephan A, Alfie A, Alamin M, Haberal M, et al. Outcome of 234 pregnancies in 140 renal transplant recipients from five middle eastern countries. Transplantation. 2008:85(6):840-3.

75. Alfi AY, Al-essawy MA, Al-lakany M, Somro A, Khan F, Ahmed S. Successful pregnancies post renal transplantation. Saudi J Kidney Dis Transpl. 2008; 19(5):746-50.

76. Oliveira LG, Sass N, Sato JL, Ozaki KS, Medina Pestana JO. Pregnancy after renal transplantation--a five-yr single-center experience. Clin Transpl. 2007; 21(3):301-4.

77. Sibanda N, Briggs JD, Davison JM, Johnson RJ, Rudge CJ. Pregnancy after organ transplantation: a report from the UK transplant pregnancy registry. Transplantation. 2007:83(10):1301-7.

78. Yassaee F, Moshiri F. Pregnancy outcome in kidney transplant patients. Urol J. 2007:4(1):14-7.

79. Kurata A, Matsuda Y, Tanabe K, Toma H, Ohta H. Risk factors of preterm delivery at less than 35 weeks in patients with renal transplant. Eur J Obstet Gynecol Reprod Biol. 2006;128(1):64-8.

80. Rahamimov R, Ben-Haroush A, Wittenberg C, Mor E, Lustig S, Gafter U, Hod M, Bar J. Pregnancy in renal transplant recipients: long-term effect on patient and graft survival. A single-center experience. Transplantation. 2006;81(5):660-4.

81. Galdo T, Gonzalez F, Espinoza M, Quintero N, Espinoza O, Herrera S,

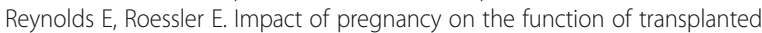
kidneys. Transplant Proc. 2005;37(3):1577-9.

82. Garcia-Donaire JA, Acevedo M, Gutierrez MJ, Manzanera MJ, Oliva E, Gutierrez E, Andres A, Morales JM. Tacrolimus as basic immunosuppression in pregnancy after renal transplantation. A single-center experience. Transplant Proc. 2005:37(9):3754-5.

83. Ghanem ME, El-Baghdadi LA, Badawy AM, Bakr MA, Sobhe MA, Ghoneim MA. Pregnancy outcome after renal allograft transplantation: 15 years experience. Eur J Obstet Gynecol Reprod Biol. 2005;121(2):178-81.

84. Pour-Reza-Gholi F, Nafar M, Farrokhi F, Entezari A, Taha N, Firouzan A Einollahi B. Pregnancy in kidney transplant recipients. Transplant Proc. 2005; 37(7):3090-2.

85. Yildirim Y, Uslu A. Pregnancy in patients with previous successful renal transplantation. Int J Gynaecol Obstet. 2005;90(3):198-202.

86. Keitel E, Bruno RM, Duarte M, Santos AF, Bittar AE, Bianco PD, Goldani JC, Garcia VD. Pregnancy outcome after renal transplantation. Transplant Proc. 2004;36(4):870-1.

87. Pezeshki M, Taherian AA, Gharavy M, Ledger WL. Menstrual characteristics and pregnancy in women after renal transplantation. Int J Gynaecol Obstet. 2004;85(2):119-25.

88. Hooi LS, Rozina G, Shaariah MY, Teo SM, Tan CH, Bavanandan S, Rosnawati $Y$. Pregnancy in patients with renal transplants in Malaysia. Med J Malaysia. 2003:58(1):27-36.

89. Queipo-Zaragoza JA, Vera-Donoso CD, Soldevila A, Sanchez-Plumed J, Broseta-Rico E, Jimenez-Cruz JF. Impact of pregnancy on kidney transplant. Transplant Proc. 2003;35(2):866-7

90. Thompson BC, Kingdon EJ, Tuck SM, Fernando ON, Sweny P. Pregnancy in renal transplant recipients: the Royal Free Hospital experience. Qjm. 2003; 96(11):837-44

91. Sgro MD, Barozzino T, Mirghani HM, Sermer M, Moscato L, Akoury H, Koren $\mathrm{G}$, Chitayat DA. Pregnancy outcome post renal transplantation. Teratology. 2002;65(1):5-9. 
92. Tan PK, Tan AS, Tan HK, Vathsala A, Tay SK. Pregnancy after renal transplantation: experience in Singapore General Hospital. Ann Acad Med Singapore. 2002;31(3):285-9.

93. Park Y, Cho J, Kim Y, Lee C, Choi H, Kim T, Kim H, Han S, Kwon H. Pregnancy outcome in renal transplant recipients: the experience of a single center in Korea. Am J Obstet Gynecol. 2001;185(6):S180

94. Kuvacic I, Sprem M, Skrablin S, Kalafatic D, Bubic-Filipi L, Milici D. Pregnancy outcome in renal transplant recipients. Int J Gynaecol Obstet. 2000;70(3):313-7.

95. Moon Jl, Park SG, Cheon KO, Kim SI, Kim YS, Park YW, Park K. Pregnancy in renal transplant patients. Transplant Proc. 2000;32(7):1869-70.

96. Ventura A, Martins L, Dias L, Henriques AC, Sarmento AM, Braga J, Pereira MC, Guimaraes S. Pregnancy in renal transplant recipients. Transplant Proc 2000;32(8):2611-2.

97. Arsan A, Guest G, Gagnadoux MF, Broyer M. Pregnancy in renal transplantation: a pediatric unit report. Transplant Proc. 1997:29(5):2479.

98. Rahbar K, Forghani F. Pregnancy in renal transplant recipients: an Iranian experience with a report of triplet pregnancy. Transplant Proc. 1997;29(7):2775.

99. Rieu P, Neyrat N, Hiesse C, Charpentier B. Thirty-three pregnancies in a population of 1725 renal transplant patients. Transplant Proc. 1997;29(5): 2459-60.

100. al Hassani MK, Sharma U, Mohsin N, Aghanashinikar P, al Maiman Y, Kumar MN, Daar AS. Pregnancy in renal transplantation recipients: outcome and complications in 44 pregnancies. Transplant Proc. 1995;27(5):2585.

101. Sabagh TO, Eltorkey MM, El Awad MA, Abed S. Outcome of pregnancy in venal transplant recipients taking cyclosporin a. J Obstet Gynaecol. 1995; 15(4):226-9.

102. Saber LT, Duarte G, Costa JA, Cologna AJ, Garcia TM, Ferraz AS. Pregnancy and kidney transplantation: experience in a developing country. Am J Kidney Dis. 1995;25(3):465-70.

103. Wong KM, Bailey RR, Lynn KL, Robson RA, Abbott GD. Pregnancy in renal transplant recipients: the Christchurch experience. N Z Med J. 1995; 108(1000):190-2.

104. Hadi HA, Stafford CR, Williamson JR, Fadel HE, Devoe LD. Pregnancy outcome in renal transplant recipients: experience at the medical College of Georgia and review of the literature. South Med J. 1986;79(8):959-64.

105. Talaat KM, Tyden G, Bjorkman U, Groth CG. Thirty successful pregnancies in organ transplant recipients: a single-center experience. Transplant Proc. 1994;26(3):1773.

106. Pahl MV, Vaziri ND, Kaufman DJ, Martin DC. Childbirth after renal transplantation. Transplant Proc. 1993;25(4):2727-31.

107. Muirhead N, Sabharwal AR, Rieder MJ, Lazarovits Al, Hollomby DJ. The outcome of pregnancy following renal transplantation--the experience of a single center. Transplantation. 1992;54(3):429-32.

108. Brown JH, Maxwell AP, McGeown MG. Outcome of pregnancy following renal transplantation. Ir J Med Sci. 1991;160(8):255-6.

109. Sturgiss SN, Davison JM. Perinatal outcome in renal allograft recipients: prognostic significance of hypertension and renal function before and during pregnancy. Obstet Gynecol. 1991;78(4):573-7.

110. O'Connell PJ, Caterson RJ, Stewart JH, Mahony JF. Problems associated with pregnancy in renal allograft recipients. Int J Artif Organs. 1989;12(3):147-52.

111. Ha J, Kim SJ, Kim ST. Pregnancy following renal transplantation. Transplant Proc. 1994;26(4):2117-8.

112. Marushak A, Weber T, Bock J, Birkeland SA, Hansen HE, Klebe J, Kristoffersen K, Rasmussen K, Olgaard K. Pregnancy following kidney transplantation. Acta Obstet Gynecol Scand. 1986;65(6):557-9.

113. O'Donnell D, Sevitz H, Seggie JL. Pregnancy after renal transplantation. Aust NZ J Med. 1985;15(3):320-5.

114. Waltzer WC, Coulam CB, Zincke $H$, Sterioff S, Frohnert PP. Pregnancy in renal transplantation. Transplant Proc. 1980;12(1):221-6.

Ready to submit your research? Choose BMC and benefit from:

- fast, convenient online submission

- thorough peer review by experienced researchers in your field

- rapid publication on acceptance

- support for research data, including large and complex data types

- gold Open Access which fosters wider collaboration and increased citations

- maximum visibility for your research: over $100 \mathrm{M}$ website views per year

At $\mathrm{BMC}$, research is always in progress.

Learn more biomedcentral.com/submissions 